Supporting Information

\title{
Luminescent and Hydrophobic Wood Films as Optical Lighting Materials
}

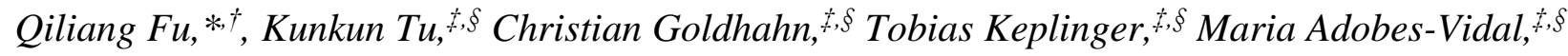
Mathias Sorieul, ${ }^{\dagger}$ and Ingo Burgert ${ }^{*}, \dot{t}, \xi$

${ }^{\dagger}$ Scion, 49 Sala Street, Private Bag 3020, Rotorua 3046, New Zealand

*Wood Materials Science, ETH Zürich, 8093 Zürich, Switzerland

${ }^{\S}$ Cellulose and Wood Materials, Empa - Swiss Federal Laboratories for Materials Science and Technology, 8600 Dübendorf, Switzerland

*E-mail: qiliang.fu@ scionresearch.com; iburgert@ethz.ch 


\section{Supporting Information}

\section{Supplemental Experiments}

Characterizations. The pore size distribution was measured with a Micromeritics ASAP 2020 (Micromeritics Instrument Corp., USA) instrument. The samples were degassed at $70{ }^{\circ} \mathrm{C}$ for 24 $\mathrm{h}$, followed by analysis at $-196{ }^{\circ} \mathrm{C}$ by $\mathrm{N}_{2}$ physisorption. The data were collected at a relative pressure between 0.1 and 0.3 . The wide-angle X-ray scattering (WAXS) measurement was carried out at the SAXS/WAXS beamline with a wavelength of $\lambda=0.685 \AA$ and detector distance $320 \mathrm{~mm}$ in the Australian Synchrotron (ANSTO, Clayton Australia). The light scattering patterns were taken using a digital camera (80D, Canon) as described in previous literature by $\mathrm{Fu}$ et $a l .{ }^{1}$ The scattering light intensity profiles were analysed using Image $\mathrm{J}$ software. Klason acid-soluble lignin content was determined by acid hydrolysis with $72 \mathrm{wt} \%$ sulfuric acid at $121^{\circ} \mathrm{C}$ according to TAPPI T222 om-83. Carbohydrate analysis was performed on a Dionex ICS-3000 anion exchange chromatograph (Dionex, USA). ${ }^{2}$ The moisture content was determined by the following equation.

Moisture content $=\left[\left(W_{a}-W_{d}\right) / W_{d}\right] \times 100 \%$

where $W_{a}$ is the sample weight when the sample was conditioned in the corresponding relative humidity until a constant value was reached, and $W_{d}$ is the dry sample weight $\left(103 \pm 2{ }^{\circ} \mathrm{C}, 24 \mathrm{~h}\right)$. The wavelength was measured on a Zeiss LSM 880 confocal equipped with a 34 channel GaAsP spectral detector and 10x 0.45 NA and 20x 0.75 NA objective lenses. Software acquisition (Zeiss Zen software) was set to lambda mode with wavelength range set to $400 \mathrm{~nm}$ to $700 \mathrm{~nm}$. Laser excitation lines used, together with the appropriate main dichroic beam splitter for each line, were $405 \mathrm{~nm}, 440 \mathrm{~nm}, 458 \mathrm{~nm}, 514 \mathrm{~nm}, 561 \mathrm{~nm}$ and $633 \mathrm{~nm}$. Regions of interest were drawn on Zen software and spectral curves plotted, and curve data were exported to a spreadsheet. 


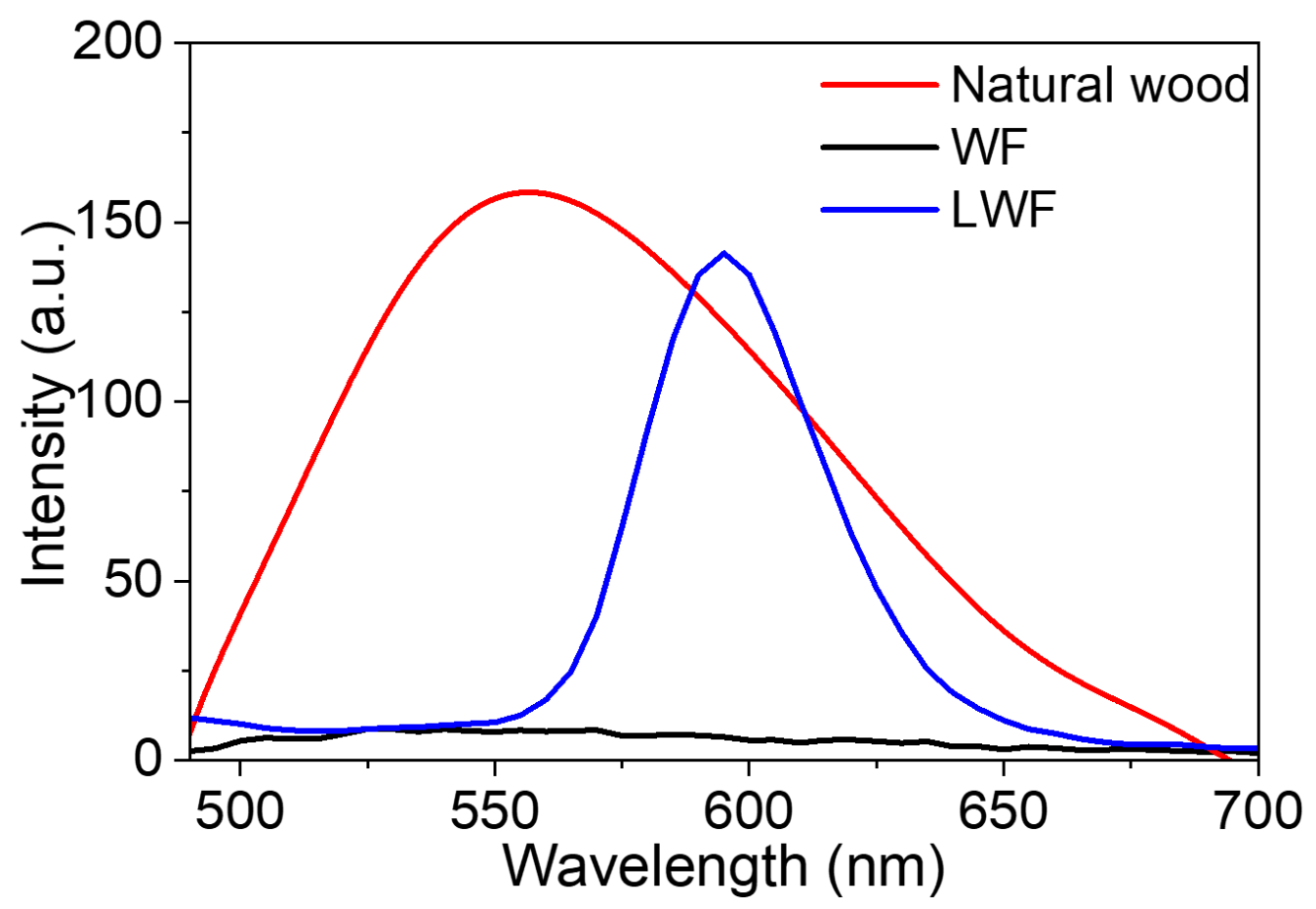

Figure S1. Fluorescence spectra of natural wood, transparent WF and LWF. 

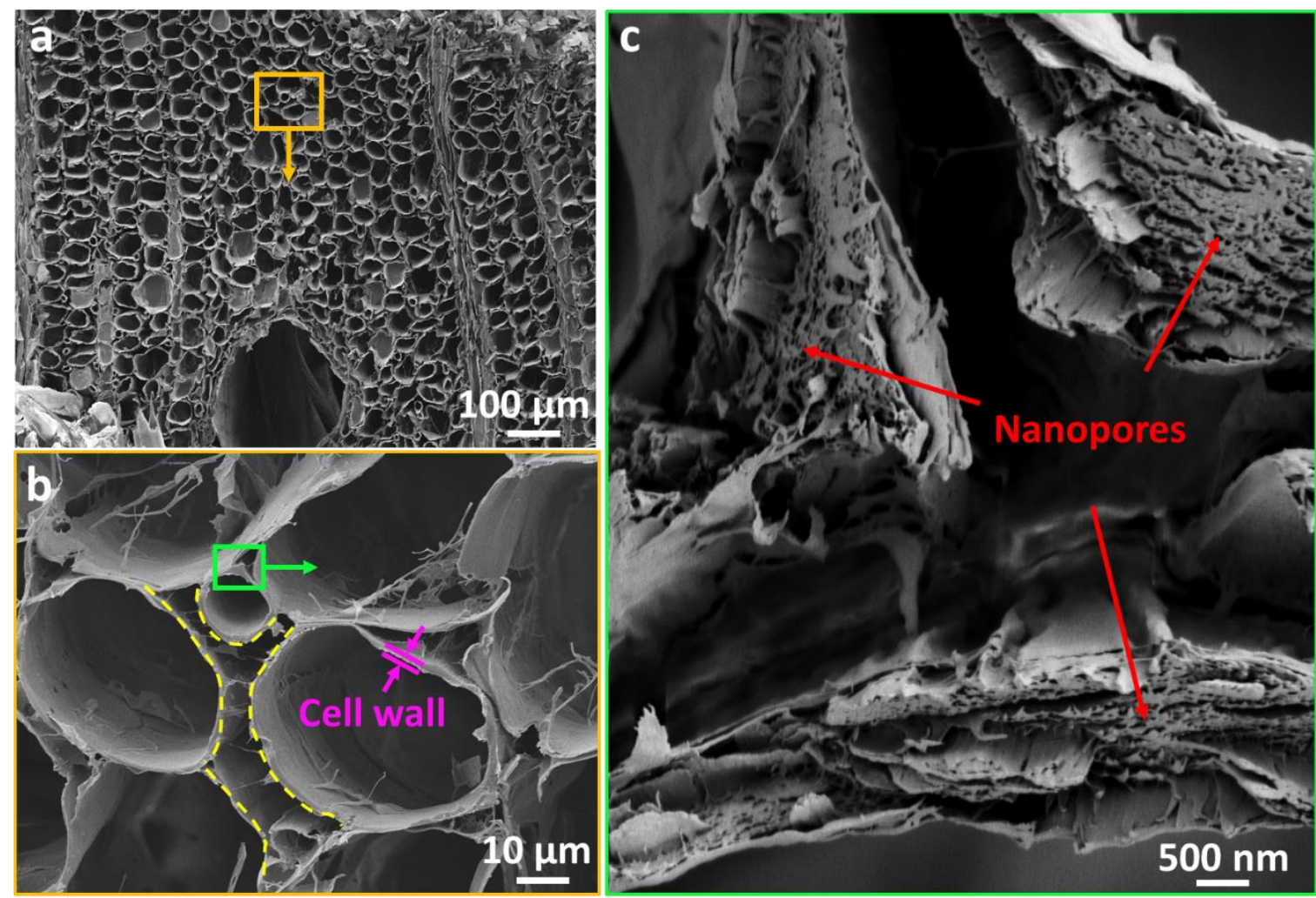

Figure S2. SEM images of treated wood. (a) Honeycomb-like structure (cross-section) of treated wood. (b) Cell wall morphology of the treated wood. The pink arrows indicate the thickness of the cell wall. The free space between the yellow dash lines suggests that some of the wood components were removed from the compound middle lamella and cell wall corners. (c) High resolution SEM image of the cell wall from the treated wood. The red arrows indicate the nanopores generated after the chemical treatment process. 


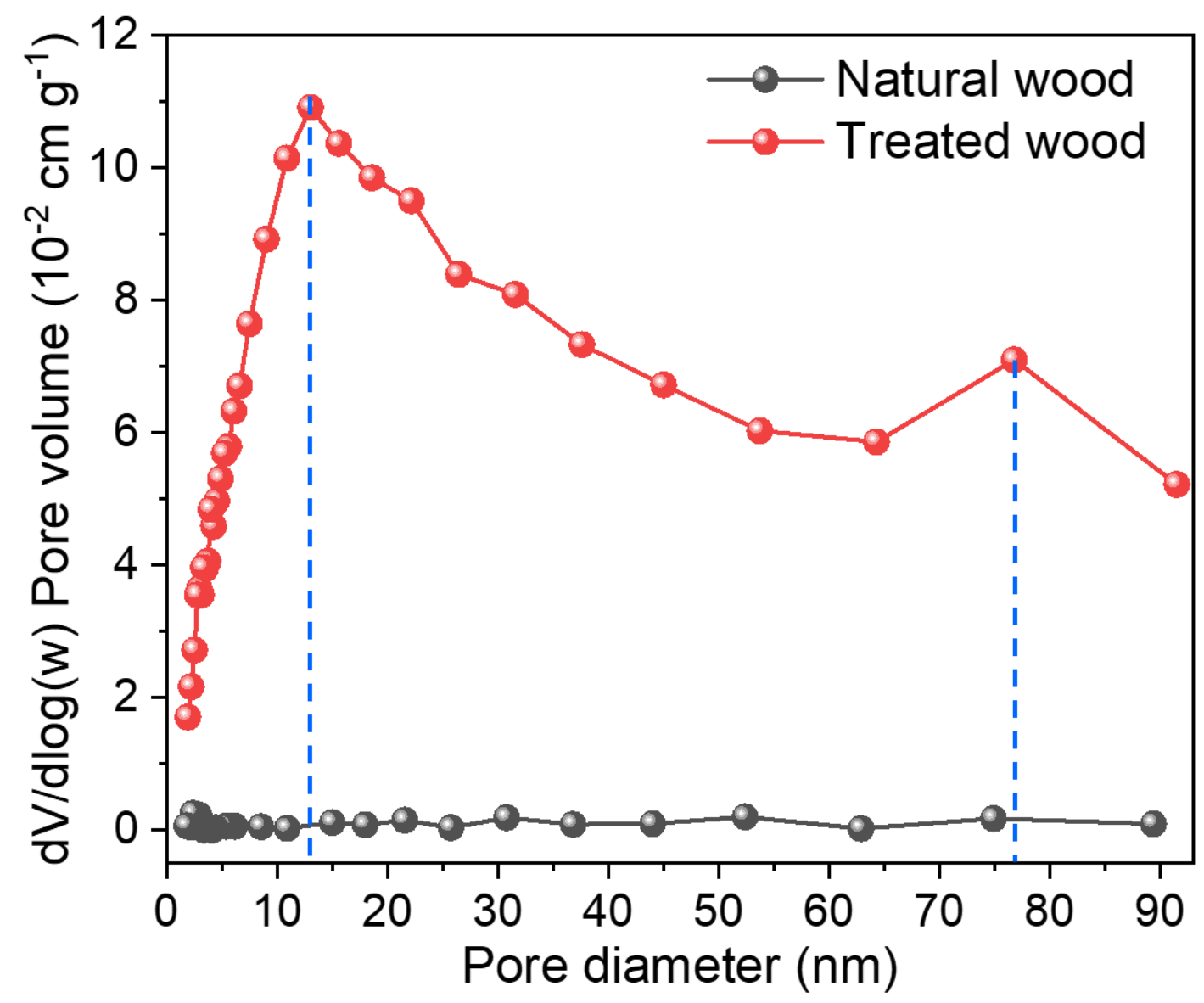

Figure S3. Pore volume distribution from BET desorption analysis for natural and treated wood, respectively. BET surface areas of natural and treated wood are $1.1 \mathrm{~m}^{2} \mathrm{~g}^{-1}$ and $46 \mathrm{~m}^{2} \mathrm{~g}^{-1}$, respectively. A large volume of pore size between $12 \mathrm{~nm}$ to $77 \mathrm{~nm}$ is shown in the curve of treated wood. 


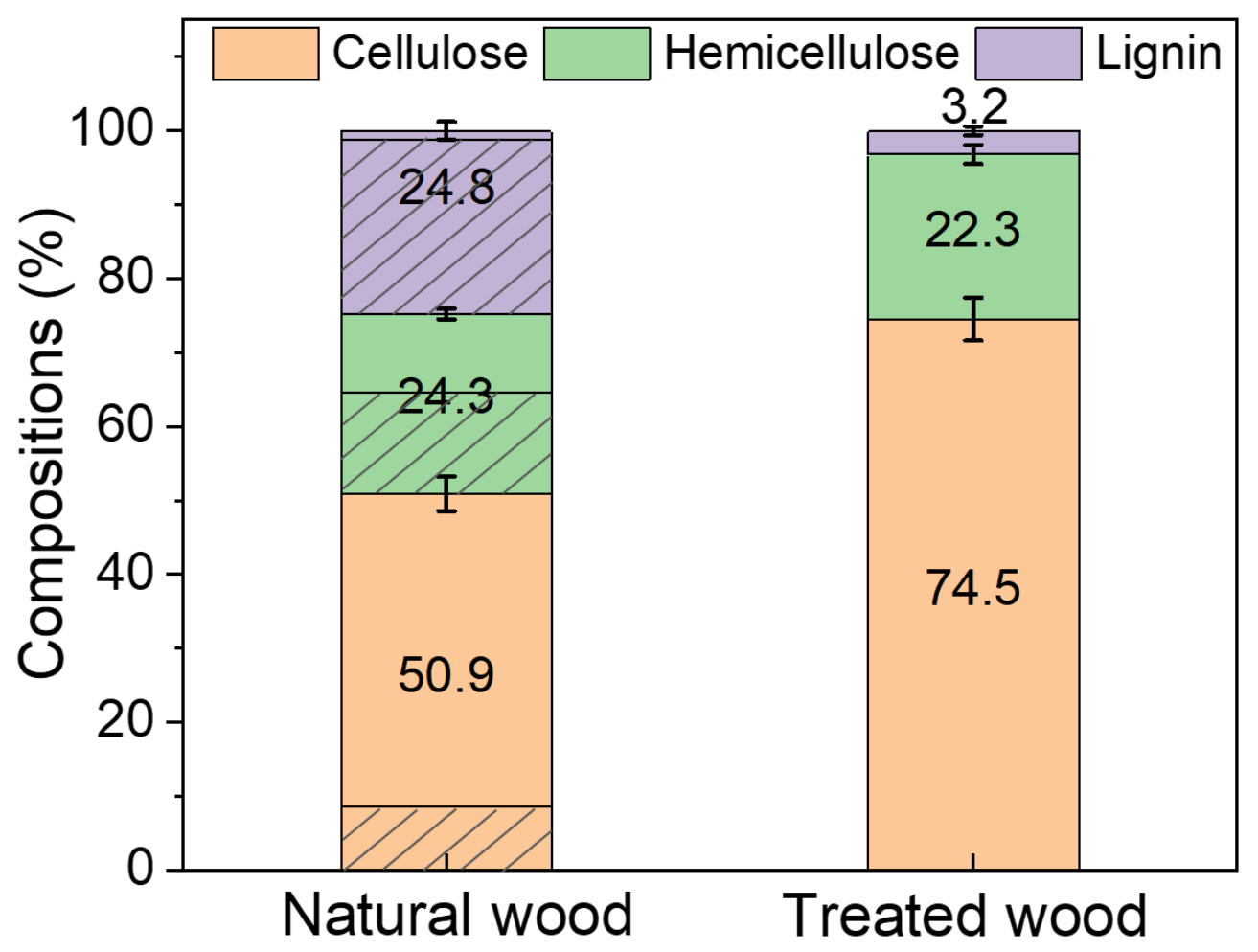

Figure S4. Chemical compositions of natural and treated wood. The composition of the treated wood is compared to that of the natural wood. The treated wood had a $45 \mathrm{wt} \%$ of mass loss (natural wood as reference) after chemical treatment. The hatched areas indicate the proportion (absolute values) of each component removed from the natural wood. 


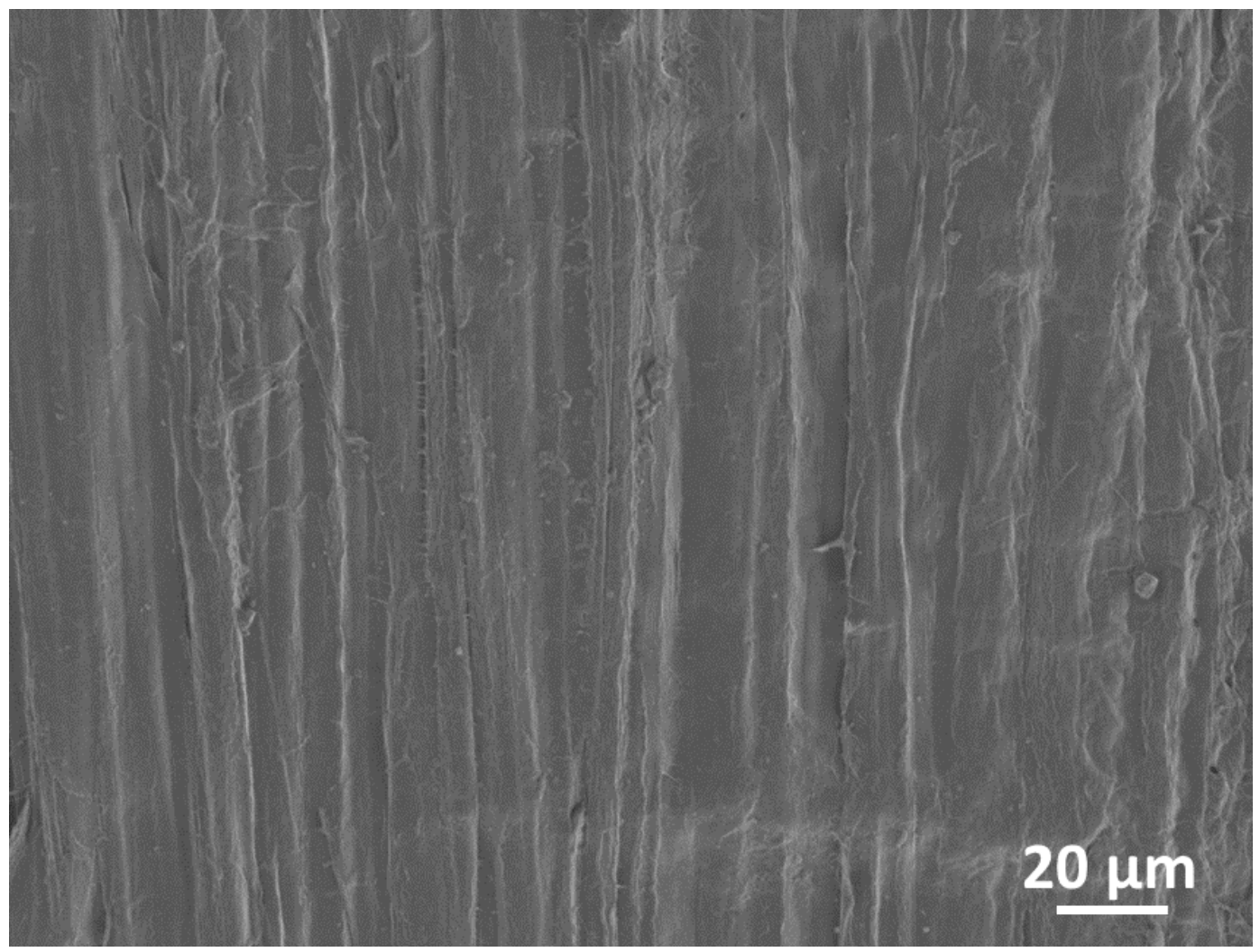

Figure S5. SEM image of the WF surface. The delignified wood fibers are exposed to the WF surface. 


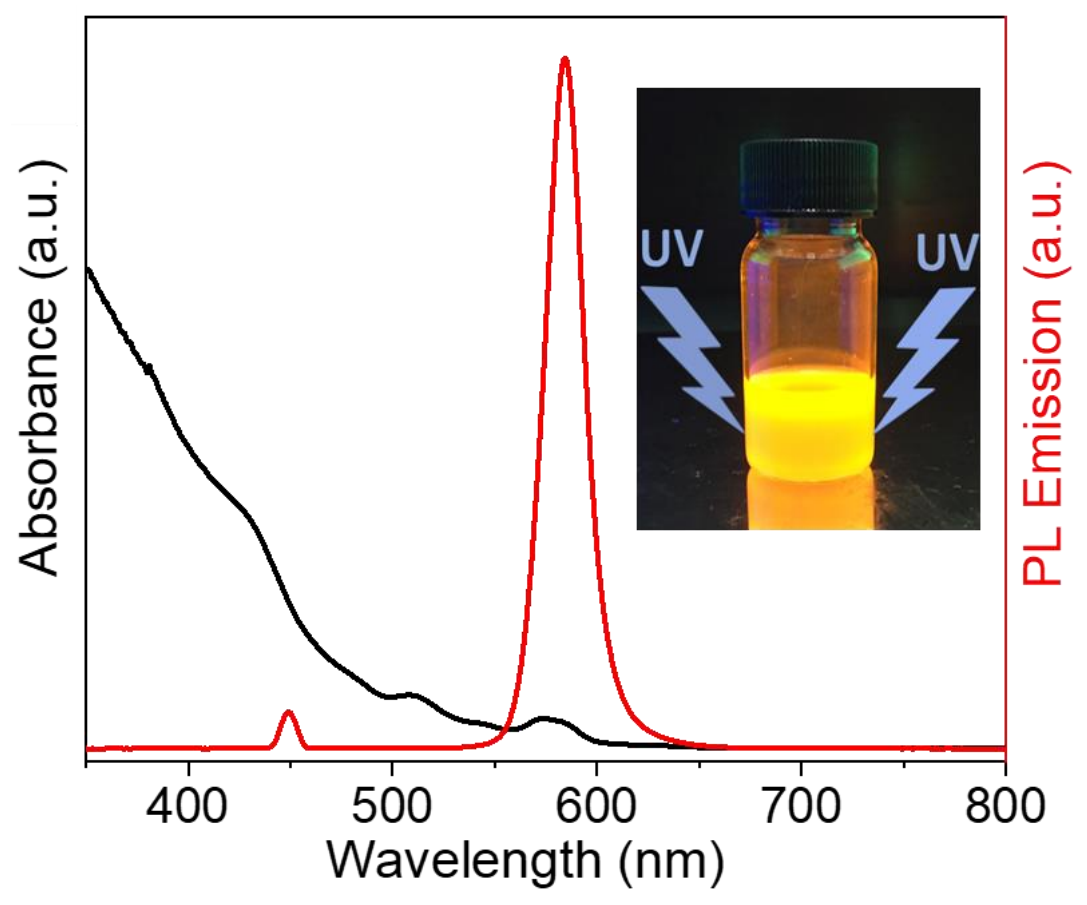

Figure S6. Luminescence and absorption spectra of the QDs solution. The wavelength of the emission light is around $585 \mathrm{~nm}$. Photograph of QDs solution (quantum yield 65\%) exposed to a UV light source (insert). 

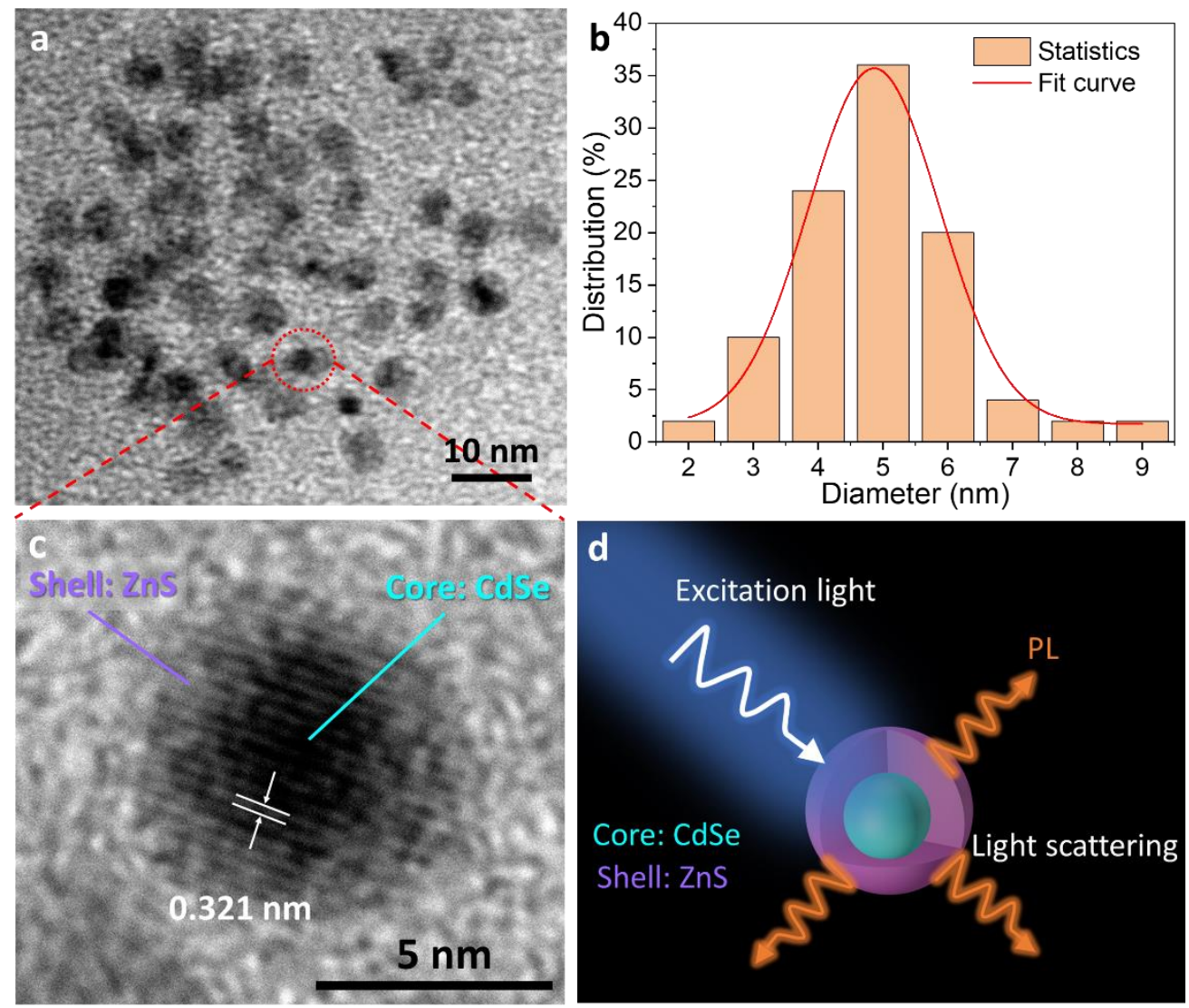

Figure S7. (a) TEM image of CdSe/ZnS QD particles. (b) Size distribution of the CdSe/ZnS QD particles $(\mathrm{n} \approx 50)$. The largest volume fraction of QD particle distribution is around $5 \mathrm{~nm}$ in diameter. (c) High resolution TEM image of a single CdSe/ZnS core-shell particle, with a crystal lattice space of $0.321 \mathrm{~nm}$ in the $\mathrm{ZnS}$ atomic plane. (d) Schematic illustration of photoluminescence (PL) and light scattering of a single $\mathrm{CdSe} / \mathrm{ZnS}$ core-shell particle. 

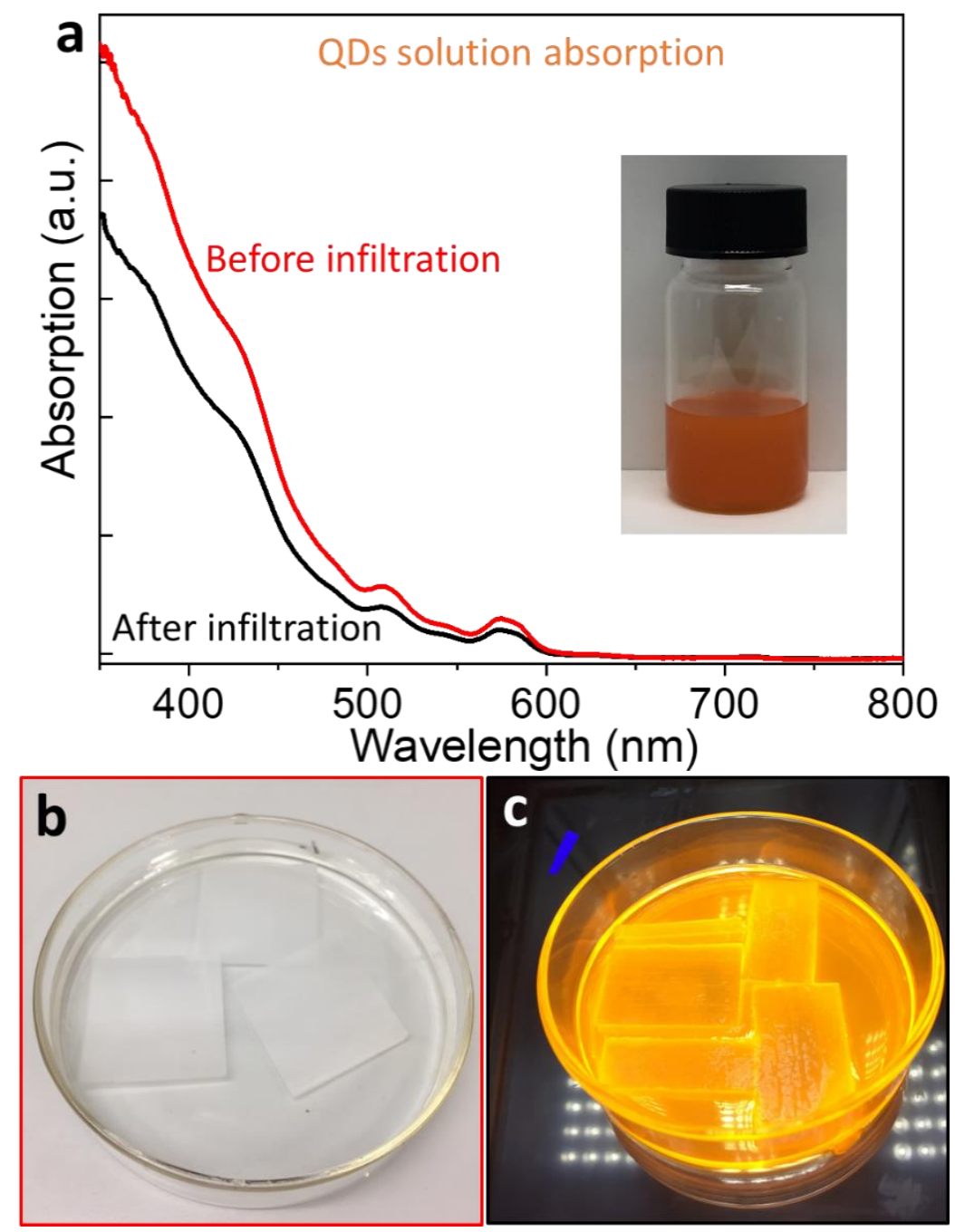

Figure S8. (a) Absorption spectra of QDs solution before (red) and after (black) impregnation. The photograph of the QD solution after infiltration (insert). The photographs of the wet treated wood sample (b) before and (c) after infiltration with the QDs solution. 


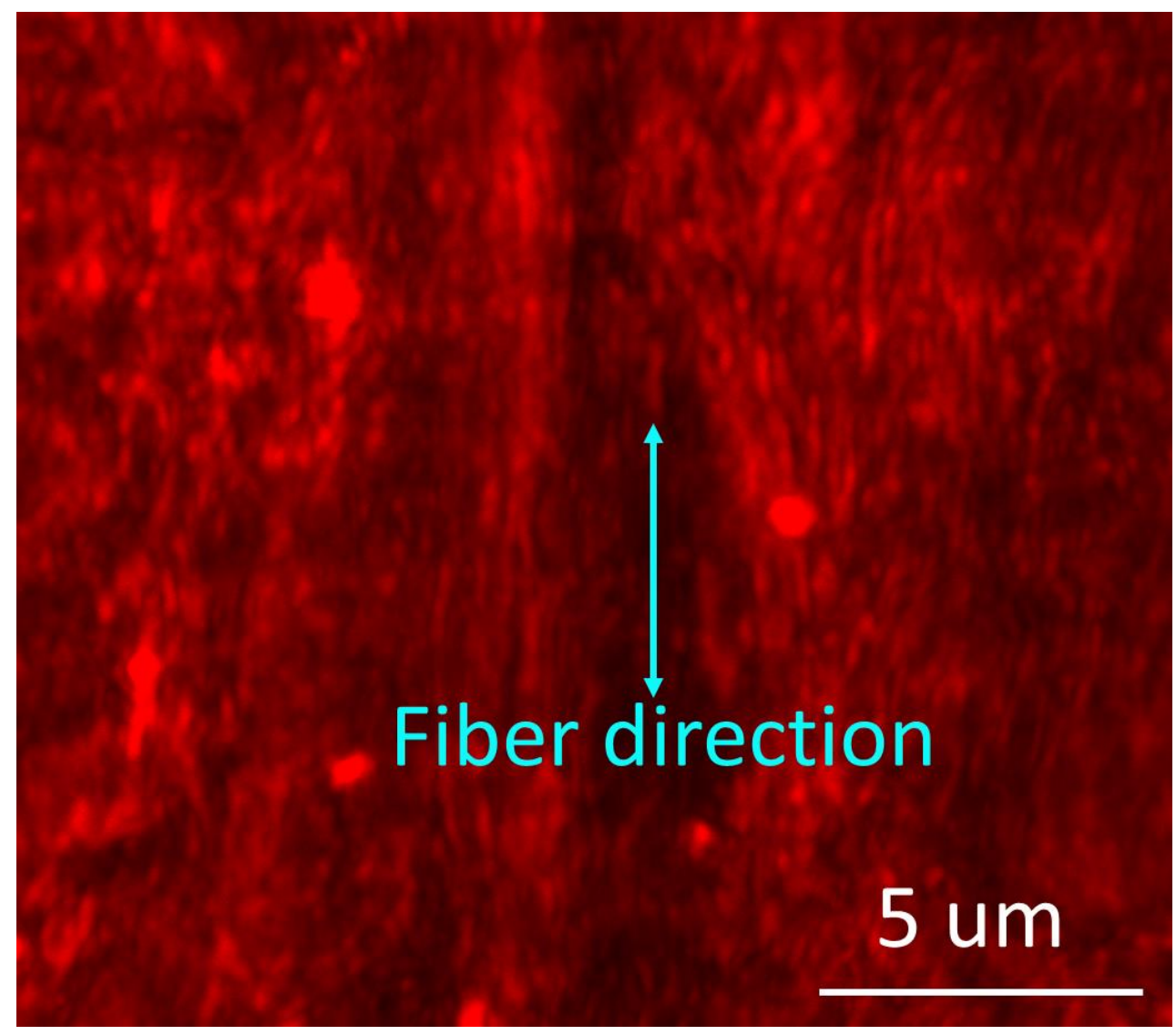

Figure S9. Fluorescence images of the LWF surface. The LWF sample was exposed to a UV light source $(v=405 \mathrm{~nm})$. 

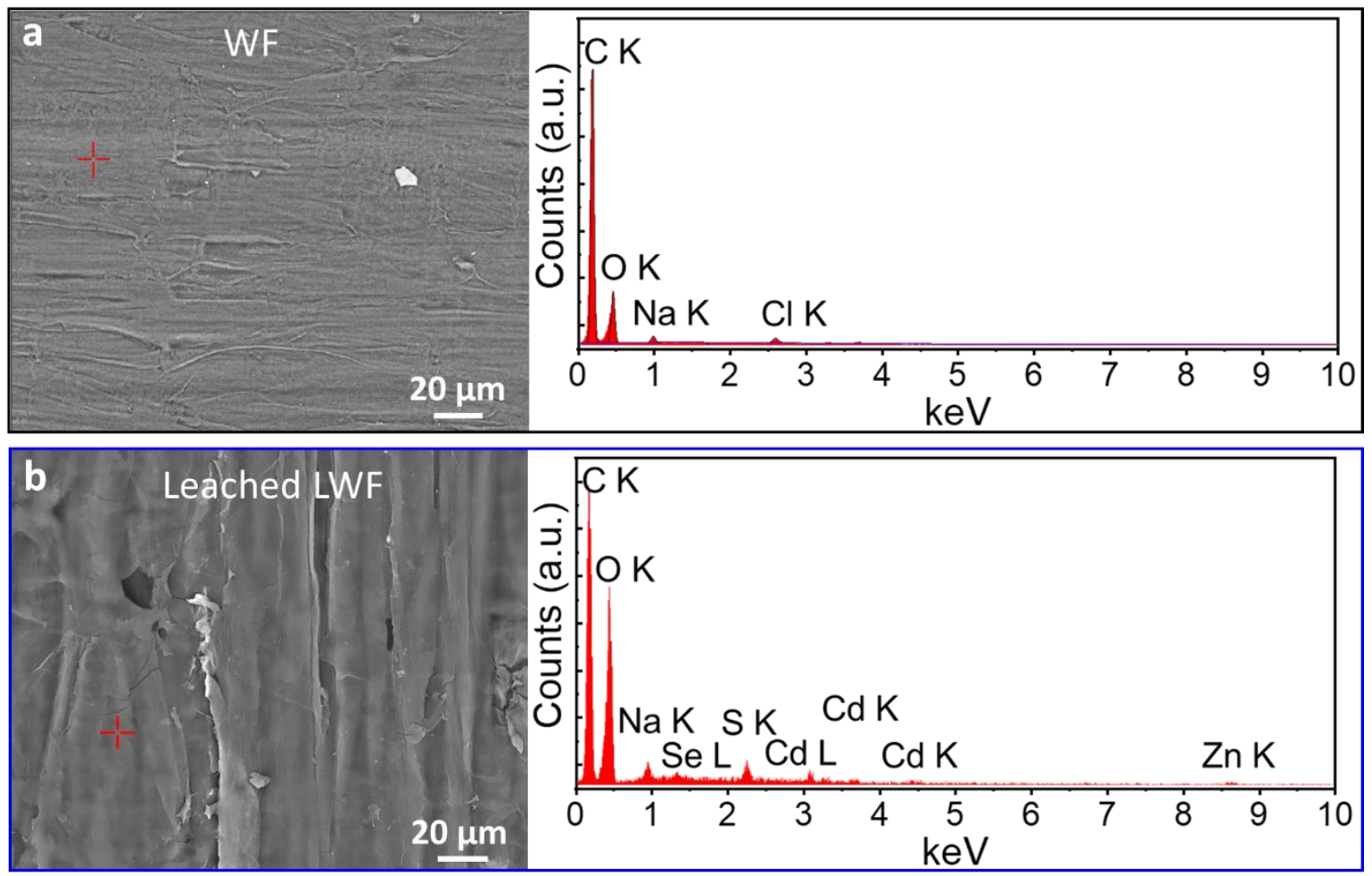

Figure S10. SEM image and EDX spectra of (a) transparent WF sample and (b) leached LWF sample; extra QD particles were removed from the leached LWF sample. 
Table S1 Element statistics of the WF, LWF and leached LWF.

\begin{tabular}{|c|c|c|c|c|c|c|c|c|}
\hline \multirow{2}{*}{ Materials } & \multicolumn{9}{|c|}{ Element (\%) } \\
\cline { 2 - 9 } & Cd L & Se L & Zn K & S K & C K & O K & Na K & Cl K \\
\hline WF & n.a. & n.a. & n.a. & n.a. & 71.6 & 27.3 & 0.7 & 0.4 \\
\hline LWF & 3.6 & 4.6 & 9.5 & 2 & 59.7 & 19.6 & 0.7 & 0.3 \\
\hline Leached & 3.5 & 3.1 & 8.4 & 2.2 & 59.3 & 22 & 0.8 & 0.7 \\
LWF & & & & & & & & \\
\hline
\end{tabular}

Note: "n.a.”: not applicable. " $a$ ": the LWF was washed in deionized water; extra QD particles were removed from leached LWF sample. $\mathrm{n}=3$ for each material. 
Table S2. Mechanical properties comparison of the traditional engineering materials, such as glass, silicon, steel, metal, alloy, polymer and plastic.

\begin{tabular}{|c|c|c|c|c|c|c|}
\hline \multirow[t]{2}{*}{ Materials } & \multirow{2}{*}{$\begin{array}{l}\text { Density } \\
\left(\mathrm{g} \mathrm{cm}^{-3}\right)\end{array}$} & \multicolumn{2}{|c|}{ Young's modulus $E$} & \multicolumn{2}{|c|}{ Strength $\sigma$} & \multirow[t]{2}{*}{ Ref. } \\
\hline & & $E(\mathrm{GPa})$ & $\begin{array}{c}\text { Specific } E \\
\left(\mathrm{GPa} \mathrm{cm}^{3} \mathrm{~g}^{-1}\right)\end{array}$ & $\sigma(\mathrm{MPa})$ & $\begin{array}{c}\text { Specific } \sigma \\
\left(\mathrm{MPa} \mathrm{cm}^{3} \mathrm{~g}^{-1}\right)\end{array}$ & \\
\hline Wood film & 1.2 & $42.7 \pm 7$ & $35.6 \pm 5.8$ & $393.8 \pm 64$ & $328.2 \pm 53.3$ & $\begin{array}{l}\text { This } \\
\text { work }\end{array}$ \\
\hline Luminescent wood film & 1.14 & $24.4 \pm 4$ & $21.4 \pm 3.5$ & $292 \pm 28$ & $256.2 \pm 24.6$ & $\begin{array}{l}\text { This } \\
\text { work }\end{array}$ \\
\hline \multicolumn{7}{|c|}{ Glass and silicon } \\
\hline Glass & 2.6 & 70 & 26.9 & 70 & 26.9 & \\
\hline Silicon & 2.4 & $130-180$ & $54.2-75$ & 165 & 68.8 & 3 \\
\hline \multicolumn{7}{|c|}{ Steels, metals and alloys } \\
\hline Stainless steel & 7.92 & 220 & 27.8 & 525 & 66.3 & \multirow{7}{*}{4} \\
\hline 4Ni-Cr-Mo steel & 7.83 & 225 & 28.7 & 1550 & 198 & \\
\hline Grey cast iron & 7.39 & 150 & 20.3 & 300 & 40.5 & \\
\hline Aluminium & 2.75 & 70 & 25.9 & 60 & 21.8 & \\
\hline Copper & 8.97 & 130 & 14.5 & 216 & 24.1 & \\
\hline $\mathrm{Mg}-6 \mathrm{Al}-1 \mathrm{Zn}$ alloy & 1.8 & 48 & 26.7 & 300 & 167 & \\
\hline 4Sn-4Al-4Mo-0.5Si alloy & 4.5 & 150 & 33.3 & 1390 & 309 & \\
\hline Fe-6.38Ti-2.4B steel & 7.4 & 236 & 31.9 & 900 & 121.6 & 5 \\
\hline $\mathrm{Al}$ alloy (2000 Series) & 2.78 & & & 496 & 178.5 & 6 \\
\hline Cr-Mn-Fe-Co-Ni alloy & 8.01 & 214.5 & 26.8 & 1280 & 159.8 & 7 \\
\hline Ti6Al4V alloy & 4.42 & 114 & 25.8 & 897 & 203 & 8 \\
\hline \multicolumn{7}{|c|}{ Polymers and plastics } \\
\hline PET & 1.34 & 2.3 & 1.7 & 55 & 41 & \\
\hline Nylon-6 & 1.1 & 2.14 & 2 & 47 & 42.7 & \\
\hline
\end{tabular}




\begin{tabular}{|c|c|c|c|c|c|}
\hline ABS & 1.1 & 2.1 & 1.9 & 34 & 30.9 \\
\hline PVC & 1.39 & 3.1 & 2.3 & 58 & 41.7 \\
\hline Polyamide-6 & 1.13 & 1 & 0.9 & 40 & 35.4 \\
\hline Polycarbonate & 1.2 & 2.1 & 1.75 & 50 & 41.7 \\
\hline Polyacrylate & 1.2 & 2.2 & 1.8 & 68 & 56.7 \\
\hline Polymethyl methacrylic & 1.18 & $2.4-3.4$ & $2.03-2.88$ & 70 & $28.6-95.2$ \\
\hline Polystyrene & 1.05 & $3-3.5$ & $2.86-3.34$ & $30-100$ & 28.8 \\
\hline Polyester & 1.39 & 9 & 6.5 & 40 & 233.3 \\
\hline Bisphenol & 1.2 & 16 & 13.3 & 280 & 28.9 \\
\hline PP & 0.9 & 2 & 2.2 & 26 & 33.7 \\
\hline HDPE & 0.95 & 1.25 & 1.3 & 32 & \\
\hline
\end{tabular}

Note: "E": Young's modulus; “ $\sigma$ ": Strength. 
Table S3. Mechanical property summary of the aligned cellulose materials, densified wood, cellulose-based composites and natural fibers.

\begin{tabular}{|c|c|c|c|c|c|c|}
\hline \multirow[t]{2}{*}{ Materials } & \multirow[t]{2}{*}{$\begin{array}{l}\text { Density } \\
\left(\mathrm{g} \mathrm{cm}^{-3}\right)\end{array}$} & \multicolumn{2}{|c|}{$\begin{array}{l}\text { Young's modulus } E \\
\text { (GPa) }\end{array}$} & \multicolumn{2}{|c|}{ Strength $\sigma(\mathrm{MPa})$} & \multirow[t]{2}{*}{ Ref. } \\
\hline & & $L$ & $T$ & $L$ & $T$ & \\
\hline Wood film & 1.2 & $42.7 \pm 7$ & $5.7 \pm 1$ & $393.8 \pm 64$ & $75.5 \pm 13$ & This work \\
\hline Luminescent wood film & 1.14 & $24.4 \pm 4$ & $5.3 \pm 0.5$ & $292 \pm 28$ & $64 \pm 7$ & This work \\
\hline $\begin{array}{l}\text { CNF film (rodman } \\
\text { distribution) }\end{array}$ & $1.08-1.53$ & $13-14.9$ & & $214-243$ & & $10-12$ \\
\hline $\begin{array}{l}\text { CNF film (stretching } \\
\text { alignment) }\end{array}$ & 1.31 & $15-33$ & & $187-576$ & & $13-15$ \\
\hline CNF fiber (stretching aligned) & $1.38-1.5$ & $\begin{array}{l}22.9- \\
65.7\end{array}$ & & $358-826$ & & 16-18 \\
\hline CNF fiber (hydrodynamic) & 1.5 & $18-86$ & & $490-1570$ & & $19-21$ \\
\hline Transparent film (top-down) & 1.31 & & & 350 & 23.2 & 22 \\
\hline $\begin{array}{l}\text { Delignified and densified } \\
\text { wood }\end{array}$ & $1.15-1.32$ & $22-35$ & & $270-773$ & 43.3 & $23-27$ \\
\hline $\begin{array}{l}\text { Delignified and densified } \\
\text { wood composite }\end{array}$ & $1.1-1.3$ & $22-70$ & & $250-670$ & & $28-30$ \\
\hline Transparent wood & $1.1-1.3$ & $1.2-3.59$ & & $23-150$ & & $2,31,32$ \\
\hline Densified wood & 1.2 & 32.9 & & 351.8 & & 33 \\
\hline $\begin{array}{c}\text { Wood film (ionic liquid } \\
\text { treated) }\end{array}$ & 1.35 & 39 & & 430 & & 34 \\
\hline Ultrathin wood & $\approx 1.3$ & 43.65 & & 342 & & 35 \\
\hline \multicolumn{7}{|c|}{ Natural fibers and bamboo } \\
\hline Cotton & $1.5-1.6$ & $5.5-12.6$ & & $281-597$ & & \\
\hline Jute & 1.3 & 26.5 & & $393-773$ & & \\
\hline Sisal & 1.5 & $9.4-22$ & & $411-635$ & & 36 \\
\hline
\end{tabular}




\begin{tabular}{|c|c|c|c|c|c|c|}
\hline Coir & 1.2 & $4-6$ & & $175-220$ & & \\
\hline Ramie & 1.5 & 61.4 & & 400 & & \\
\hline Hemp & 1.48 & 70 & & 690 & & 37 \\
\hline Bamboo & 0.8 & 19.2 & & 298 & & \\
\hline Densified bamboo & 1.35 & 75 & & 1008 & & 39 \\
\hline $\begin{array}{c}\text { Self-densified wood film } \\
\text { (TEMPO treatment) }\end{array}$ & 1.32 & 51.1 & & 449.1 & & 39 \\
\hline
\end{tabular}

Note: " $L$ ": parallel to fibre growth direction; " $T$ ": perpendicular to fibre direction; "CNF": cellulose nanofibrils 


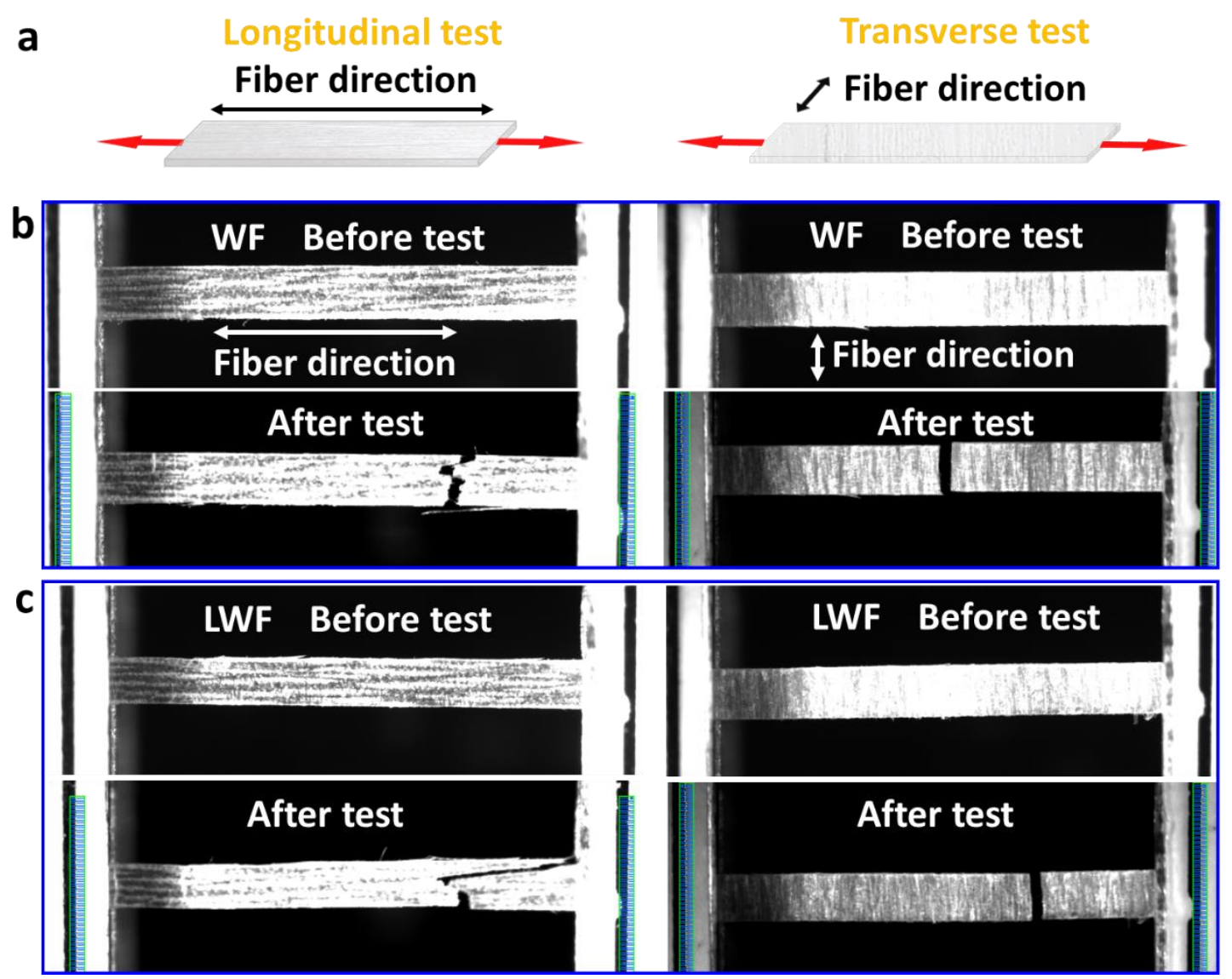

Figure S11. (a) Schematic illustration of the micro tensile test in longitudinal and transverse directions. Photographs of the micro tensile test fracture profile of (b) WF and (c) LWF samples in longitudinal and transverse directions. In longitudinal direction, the WF fracture profile of the tensile test exhibits an irregular shape with a jagged breakage line. By contrast, the fracture profile in transverse direction shows a straight breakage line. These results are similar to the test for LWF sample. 

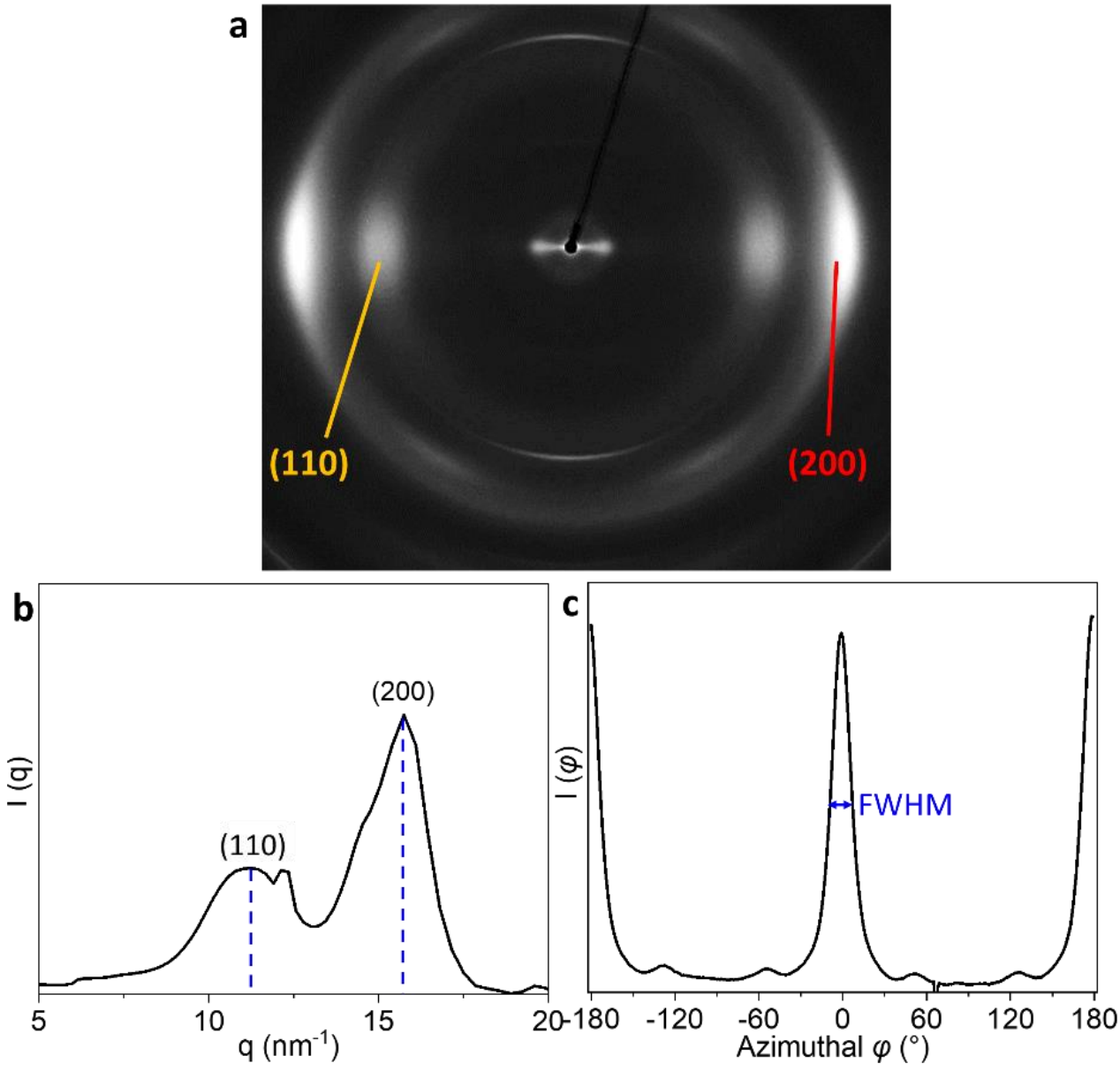

Figure S12. (a) X-ray synchrotron wide-angle X-ray scattering (WAXS) diffraction pattern of the WF with typical cellulose crystal planes (200) and (110). (b) Radial integration of the diffractogram extracted from X-ray synchrotron WAXS diffractogram (a). (c) Azimuthal integration of the (200) scattering plane from X-ray synchrotron WAXS diffractogram (a). Orientation factor. Radial integration of the diffractogram and azimuthal integration of the (200) scattering diffractogram plane at $q$ range $15.7 \pm 0.1 \mathrm{~nm}^{-1}$ are extracted from X-ray synchrotron WAXS diffractograms. The orientation factor $(f)$ is calculated and assessed the degree of cellulose orientation by the following equation, $f=(180-F W H M) / 180$ FWHM is the full width at half maximum, using Gaussian peak fitting of the X-ray synchrotron azimuthal integration curve. The cellulose crystallinity $(78 \%)$ and orientation index $(\approx 0.9)$ are calculated based on this X-ray diffraction results. 


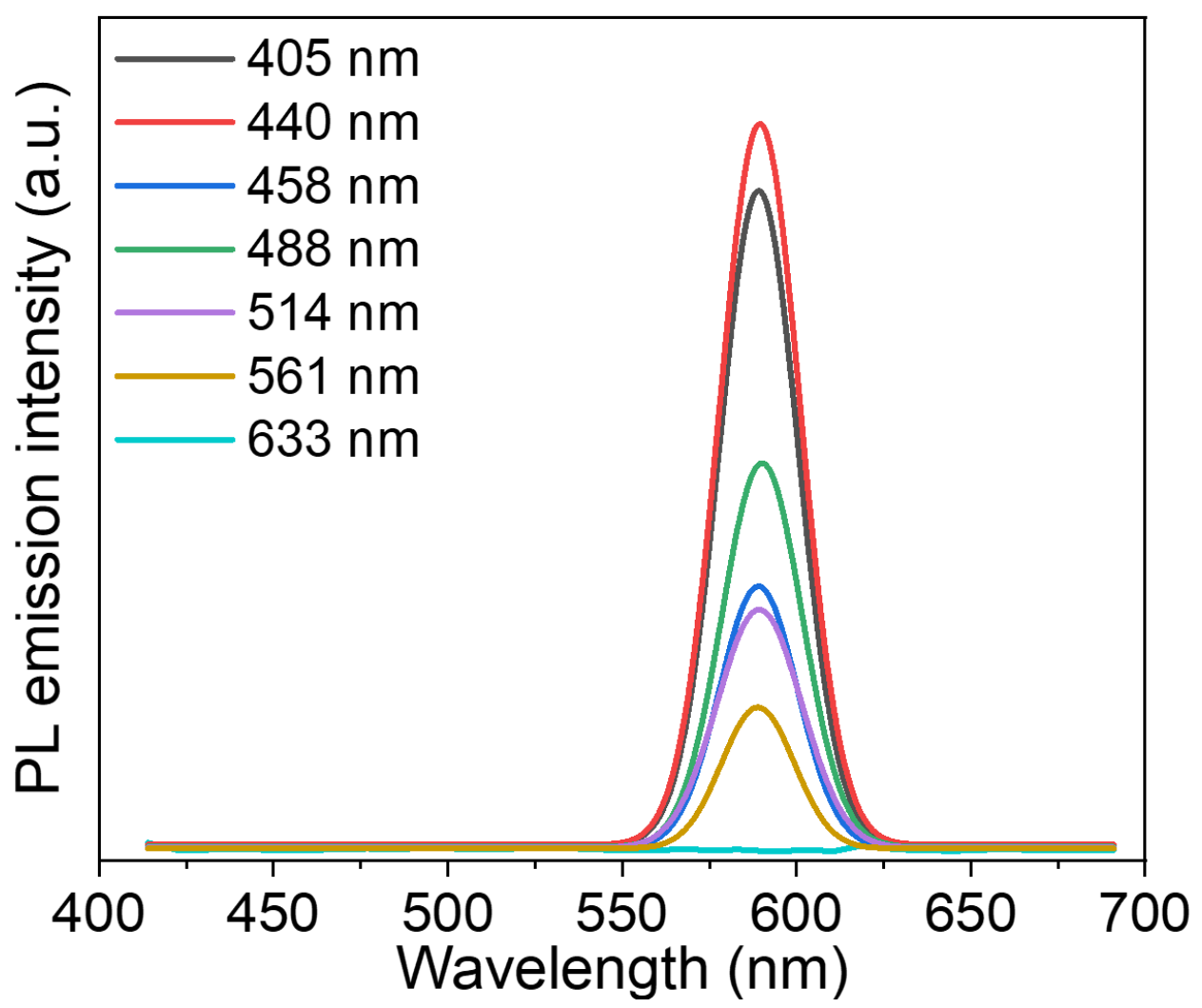

Figure S13. The emission light at different excitation wavelength from $405 \mathrm{~nm}$ to $633 \mathrm{~nm}$. 


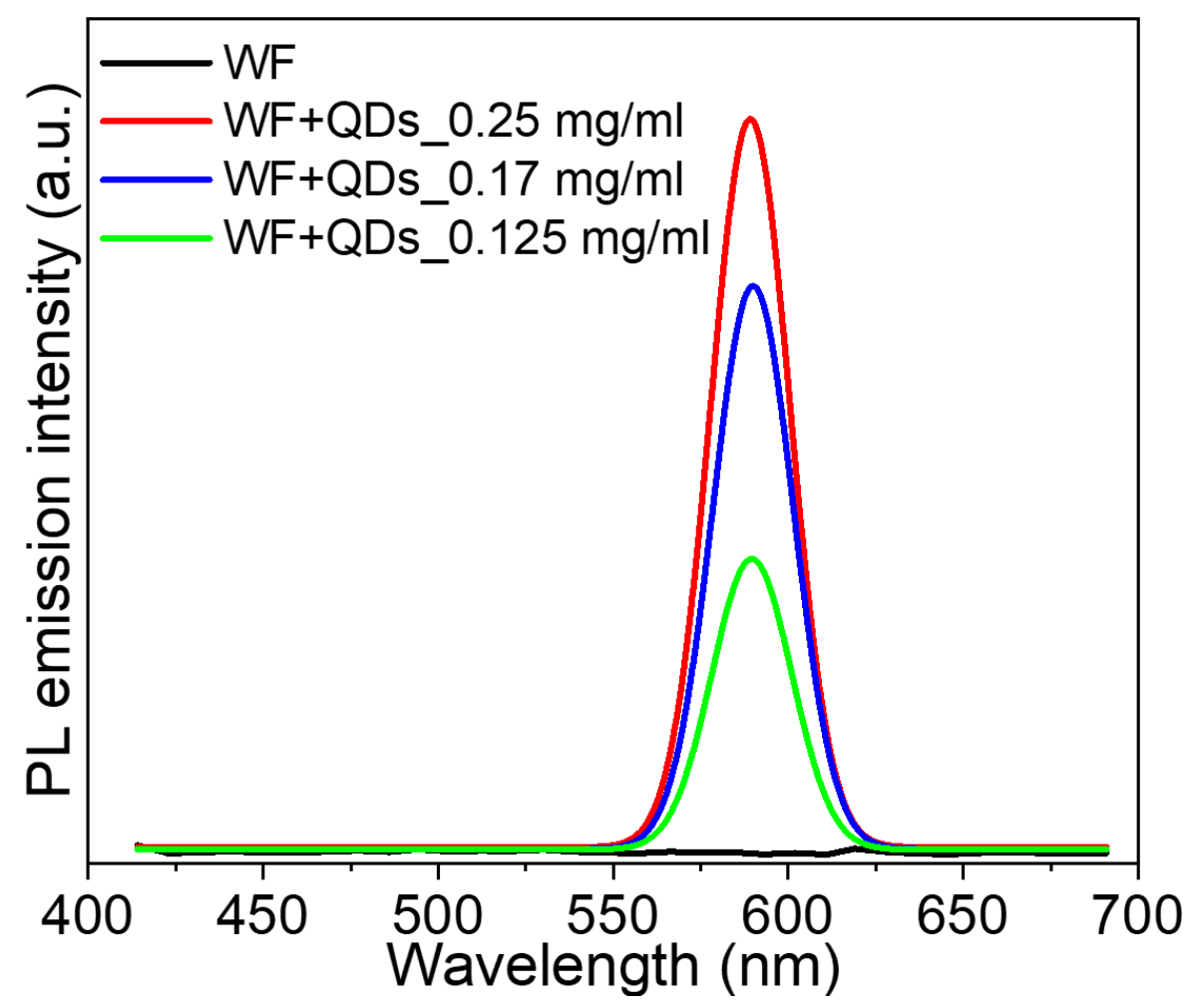

Figure S14. The photoluminescent spectra of the LWF with $0.25 \mathrm{mg} / \mathrm{mL}, 0.17 \mathrm{mg} / \mathrm{mL}$ and 0.125 $\mathrm{mg} / \mathrm{mL}$ concentration QD solutions, respectively. 


\section{$155^{\circ} \mathrm{C} 4 \mathrm{~h} \quad \mathrm{CVD}$ treatment}

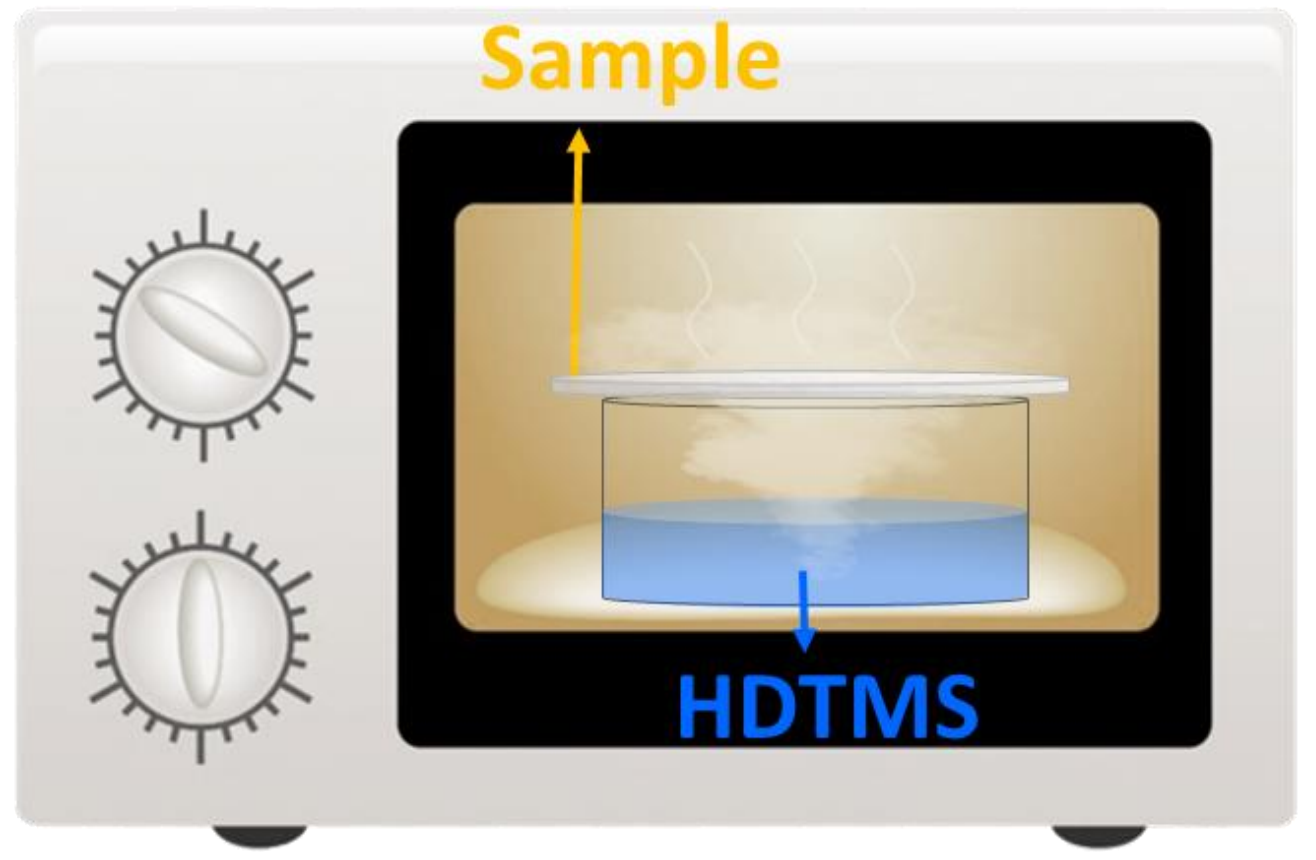

Figure S15. The schematic illustration of hydrophobic coating with HDTMS via CVD treatment. 


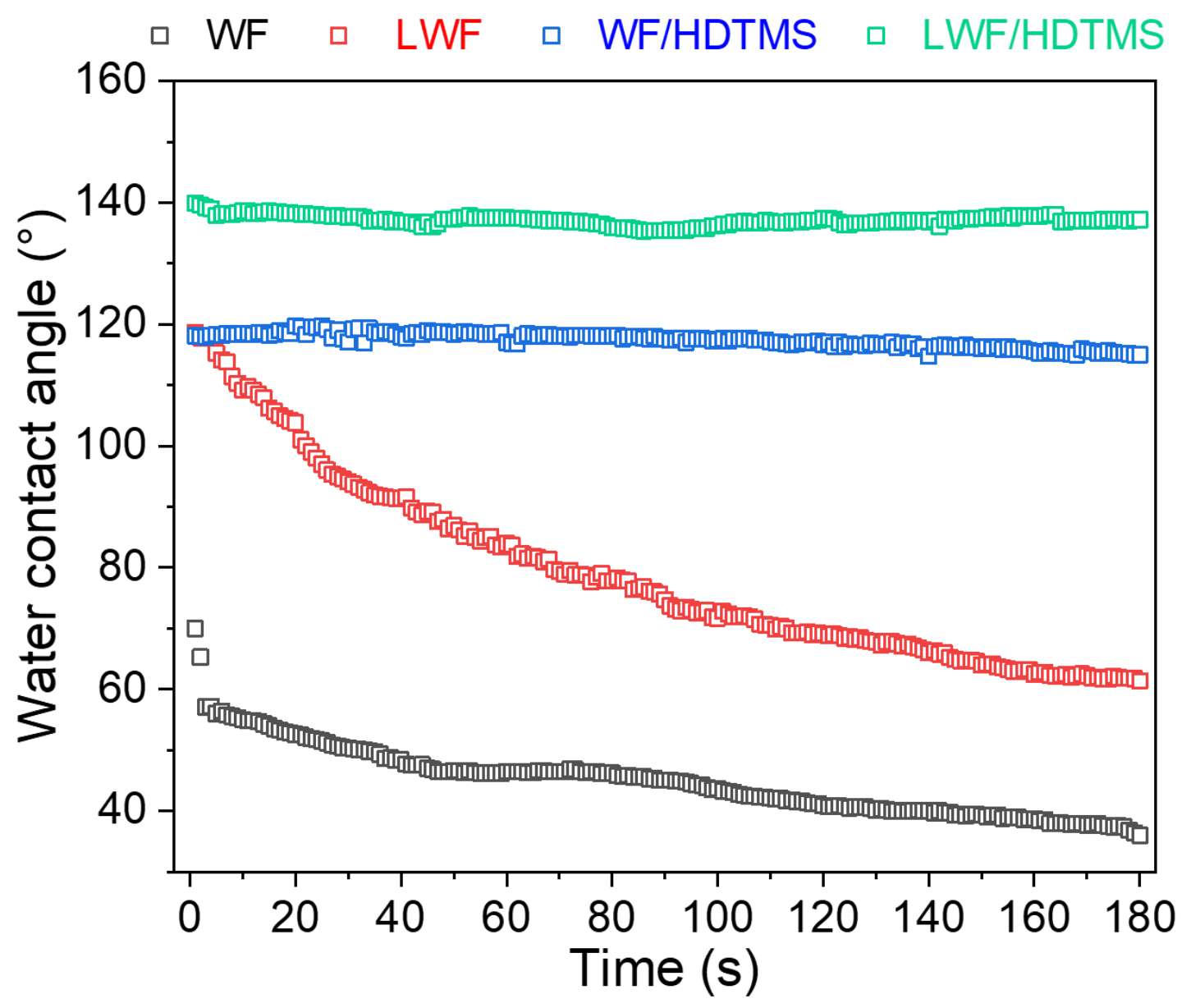

Figure S16. Dynamic water contact angle of WF, LWF, WF/HDTMS and LWF/HDTMS, respectively. 

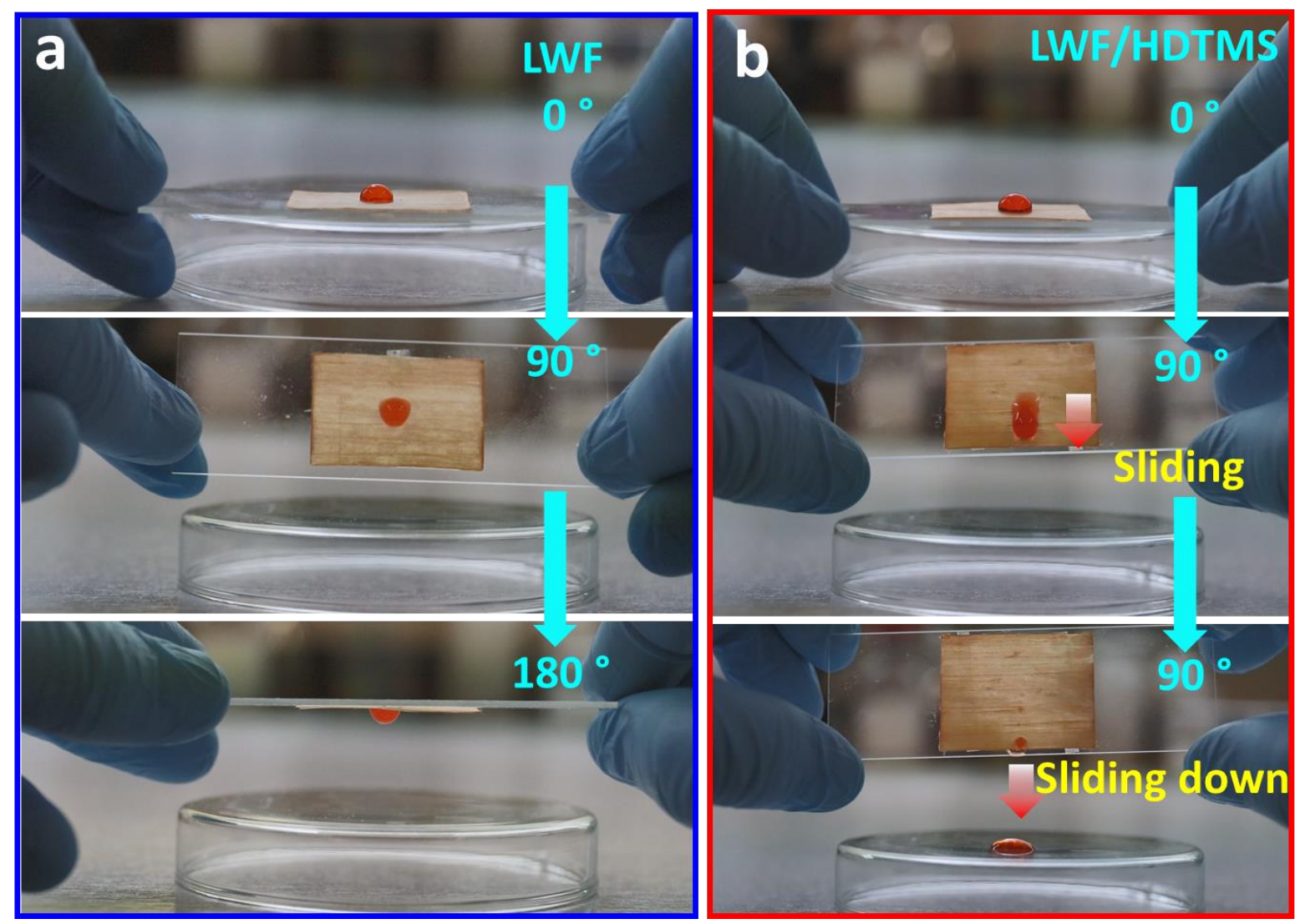

Figure S17. (a) Photographs of a water droplet (dyed with red cochineal extract) on the LWF substrate rotated through $0^{\circ}, 90^{\circ}$ and $180^{\circ}$, respectively. (b) Photographs of a dyed water droplet on the LWF/HDTMS substrate rotated through $0^{\circ}$ and $90^{\circ}$. 


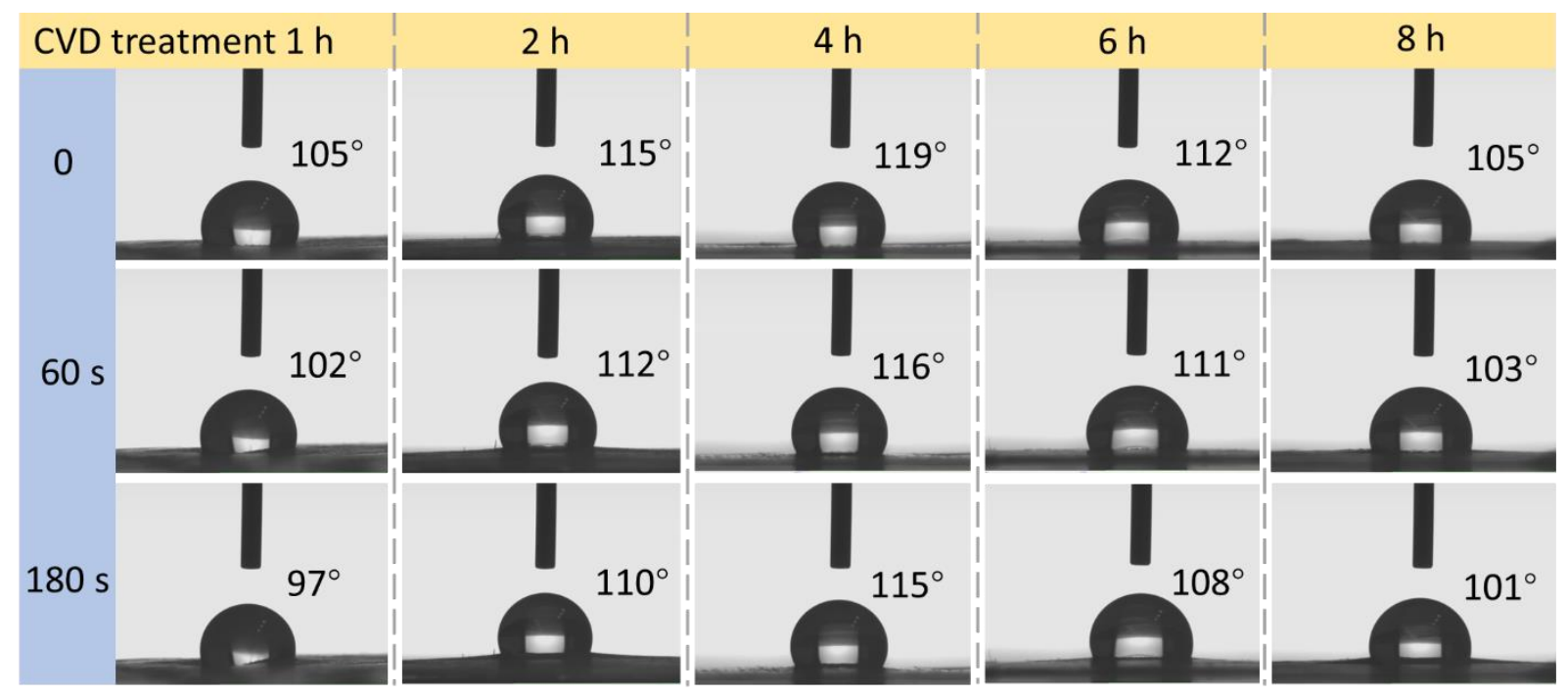

Figure S18. Water contact angles collected from a $5 \mu \mathrm{L}$ water droplet on WF/HDTMS samples after different CVD treatment time (1-8 h). 


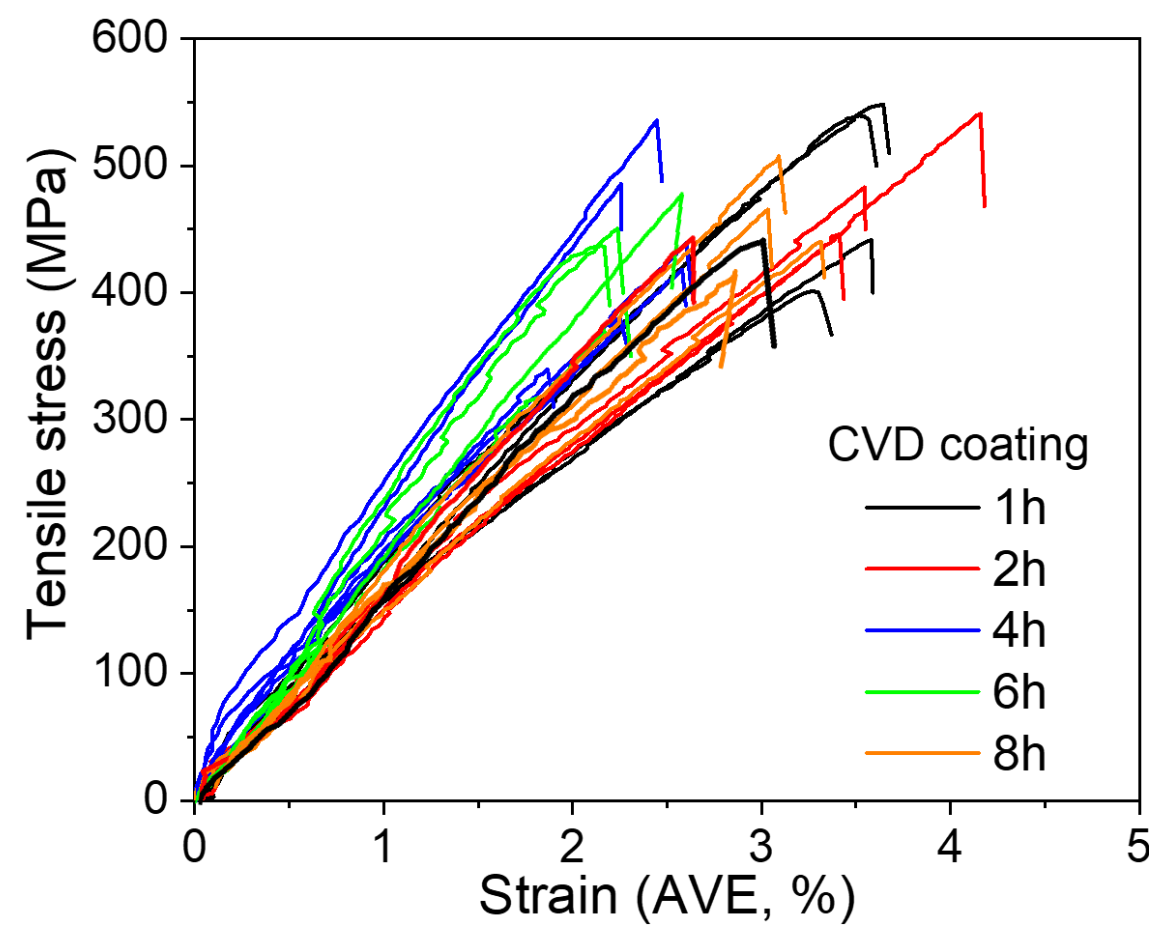

Figure S19. The tensile stress-strain curves of WF/HDTMS samples after different CVD treatment time $(1-8 \mathrm{~h})$. All sample tests were performed at $23{ }^{\circ} \mathrm{C}$ and $\mathrm{RH} 50 \%$. 


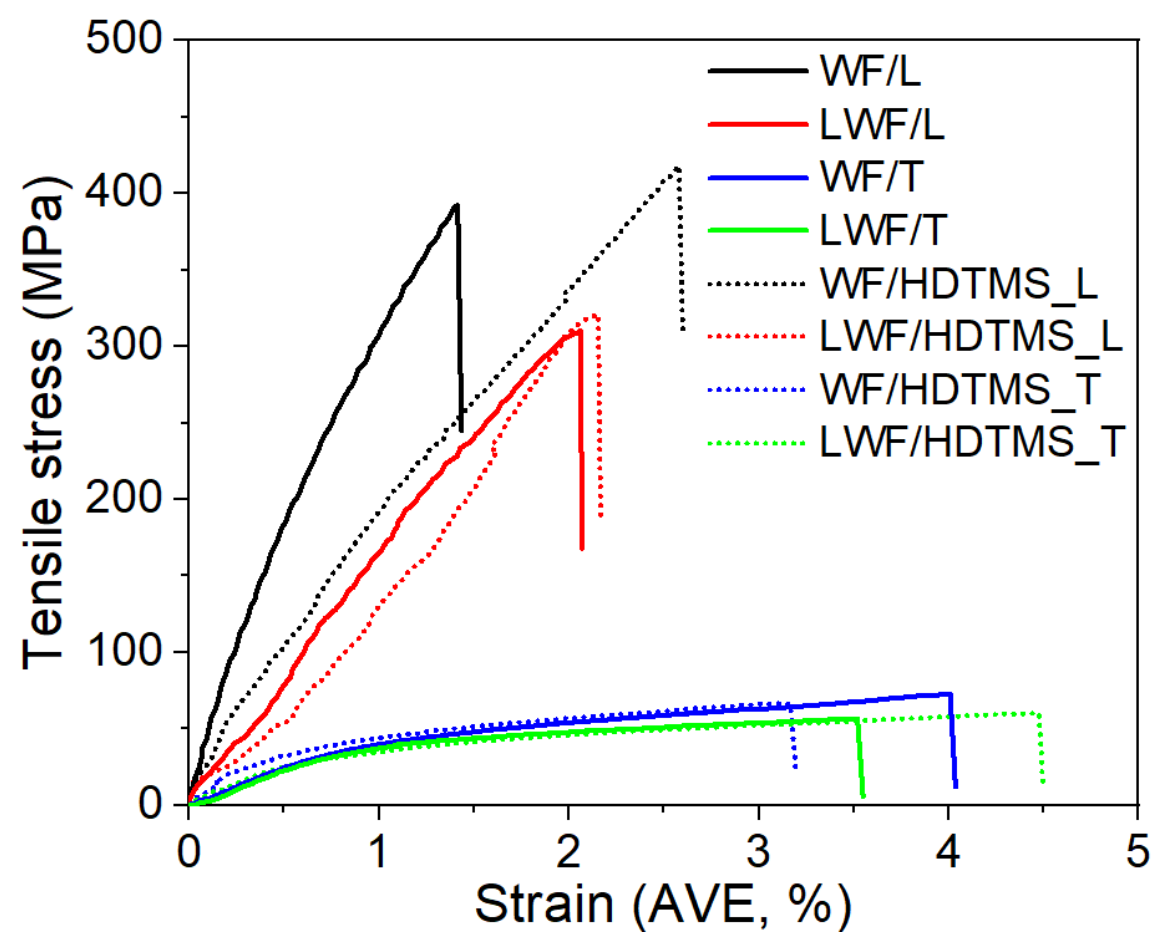

Figure S20. Tensile stress of WF and LWF samples with and without HDTMS coating in $L$ and $T$ directions. All sample tests were performed at $23{ }^{\circ} \mathrm{C}$ and $\mathrm{RH} 50 \%$. 

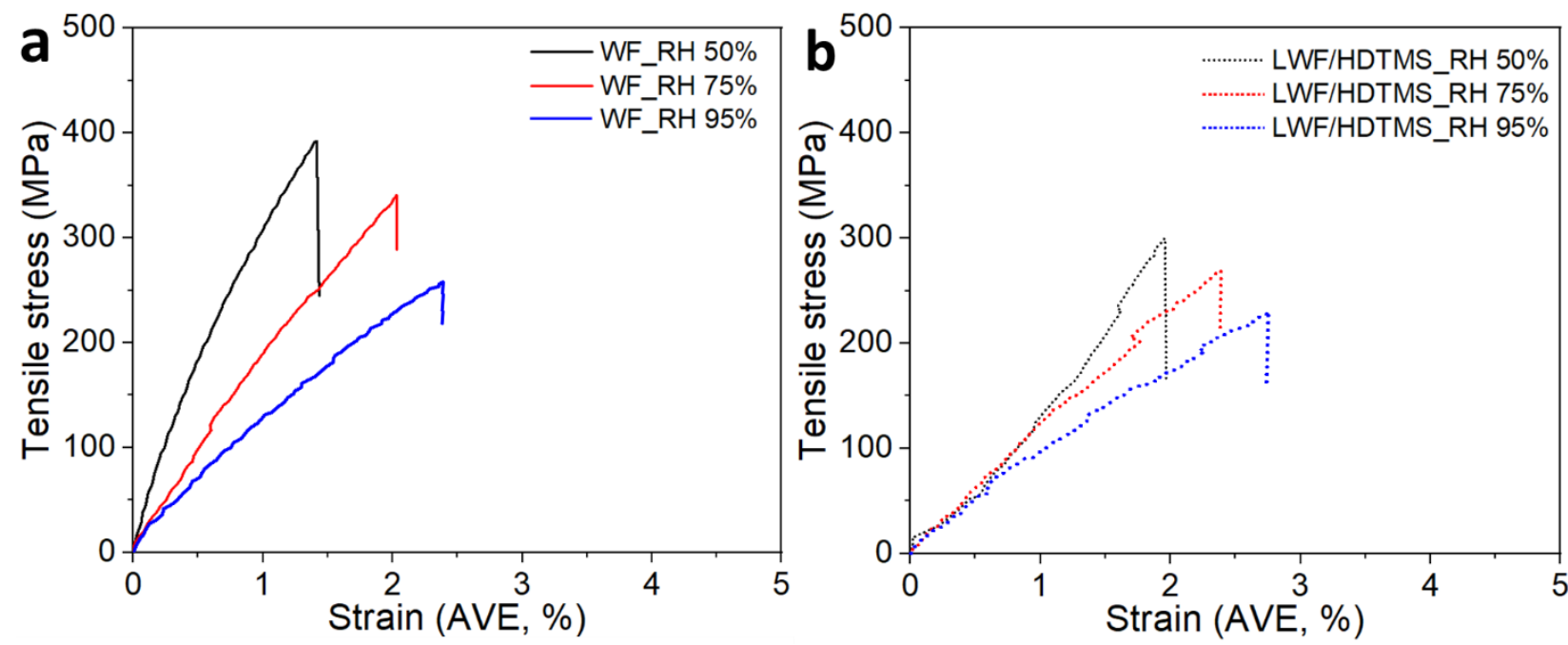

Figure S21. The tensile stress-strain curves of (a) WF and (b) LWF/HDTMS tested at different relative humidity levels $\left(50 \%, 75 \%\right.$ and $\left.95 \% ; 23{ }^{\circ} \mathrm{C}\right)$. 


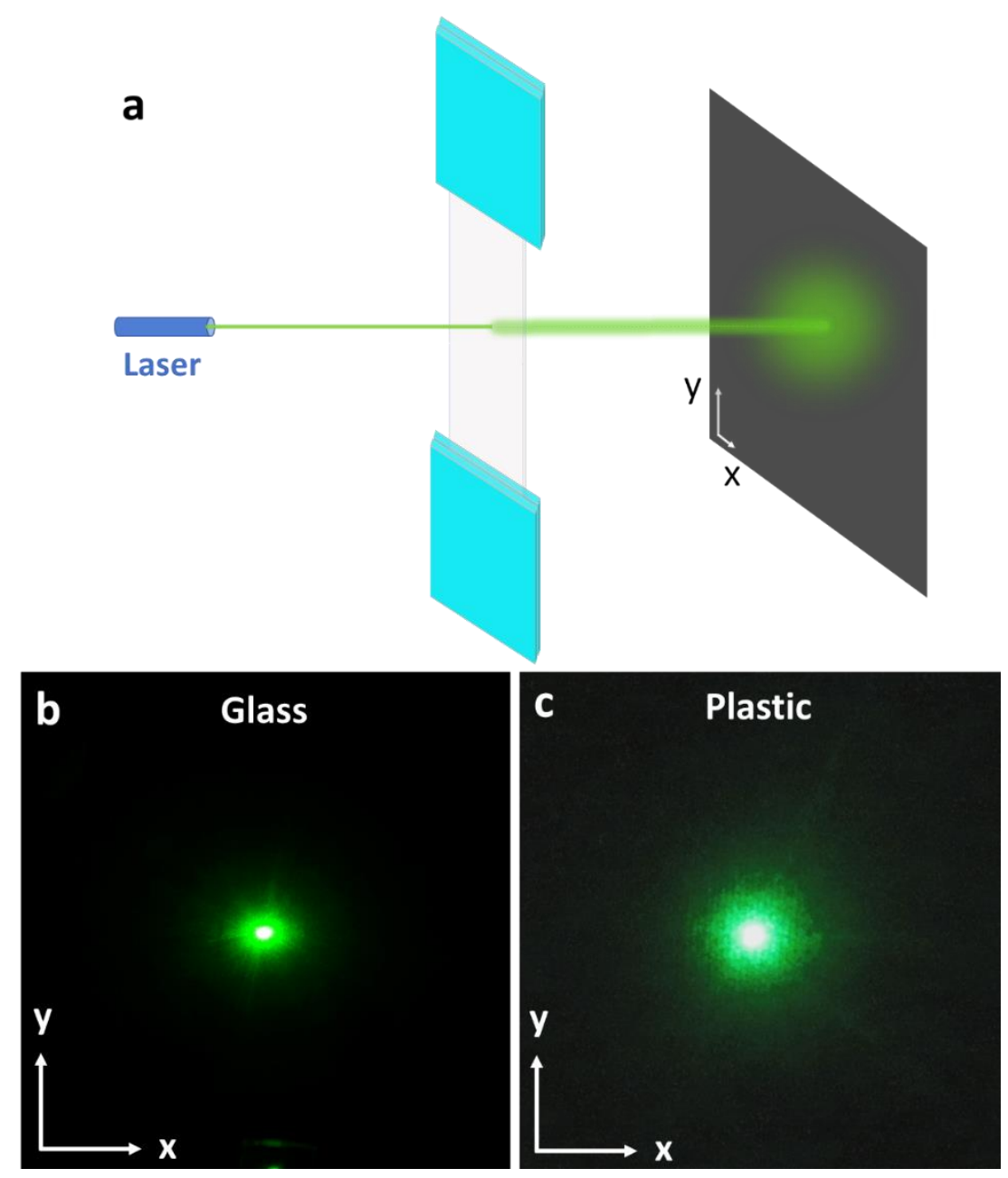

Figure S22. (a) Schematic illustration of the light scattering experiment. The light scattering patterns are collected after transmission through transparent (b) glass and (c) plastic. 


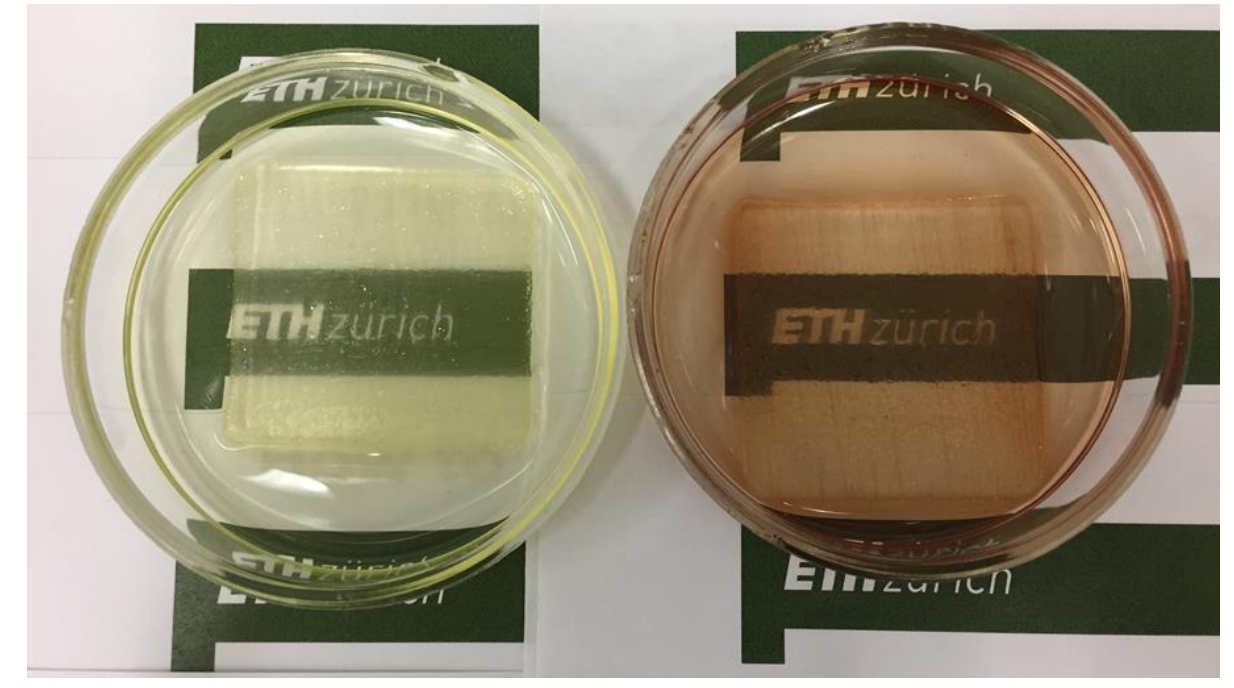

Figure S23. Photographs of transparent and gel-like treated wood/QDs under visible light. The "ETH" logo is used with permission from ETH Zürich, Switzerland. 

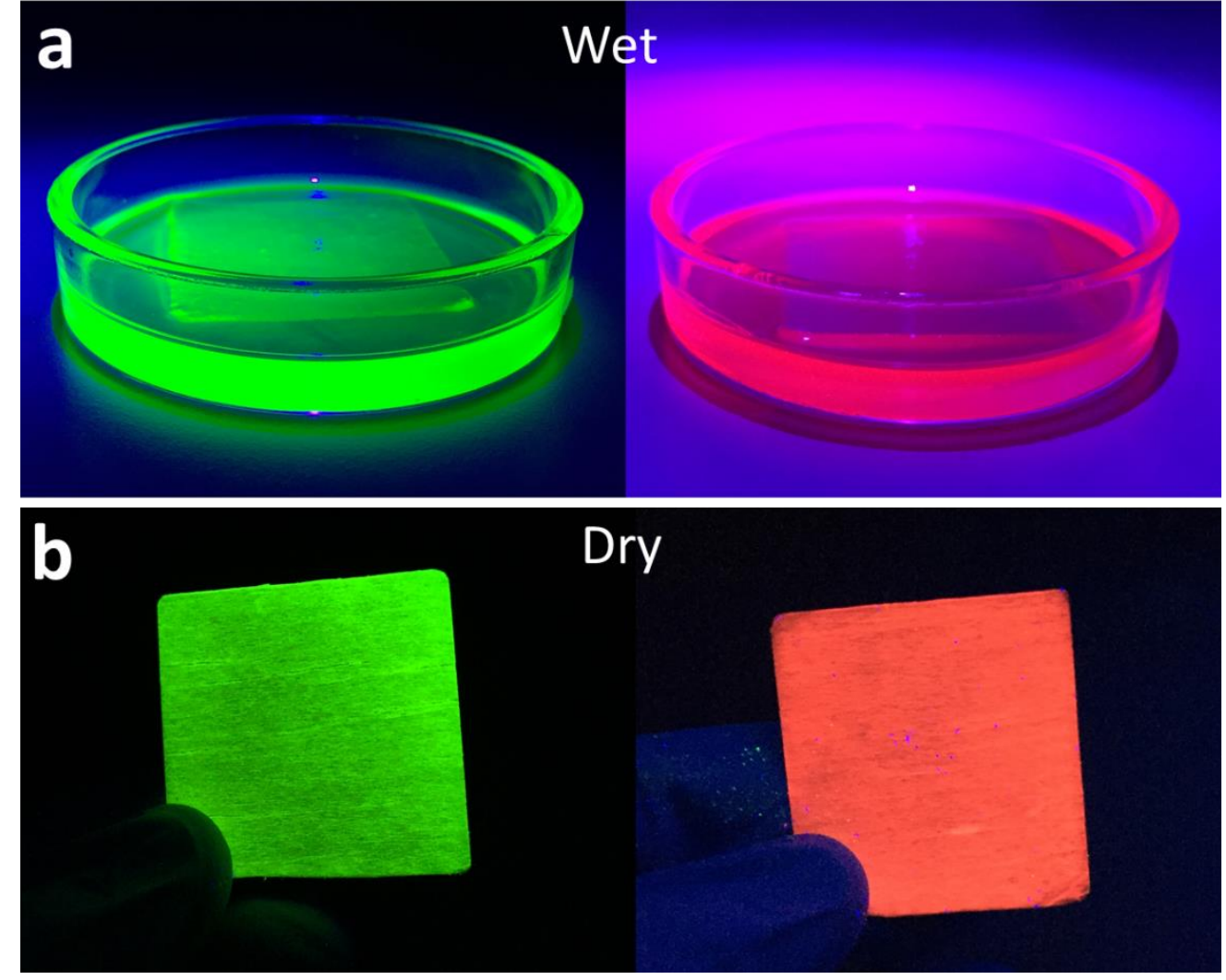

Figure S24. Photographs of LWF samples (a) at a swollen and wet stage and (b) after densification and dehydration. 


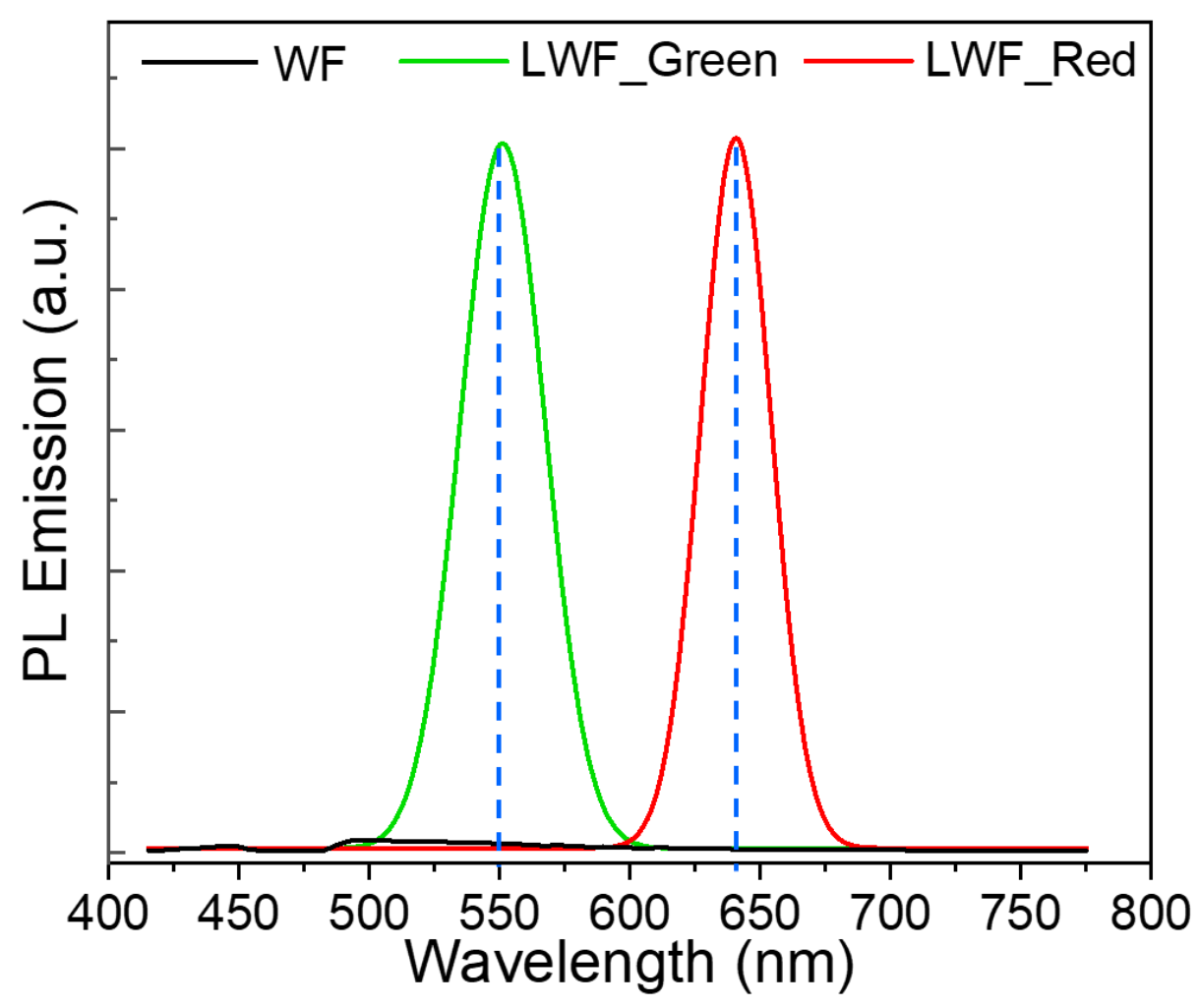

Figure S25. Photoluminescent emission spectra of the WF and LWF samples with green $\left(\lambda_{\text {emi }} \approx\right.$ $550 \mathrm{~nm})$ and red $\left(\lambda_{e m i} \approx 640 \mathrm{~nm}\right)$ colour. 


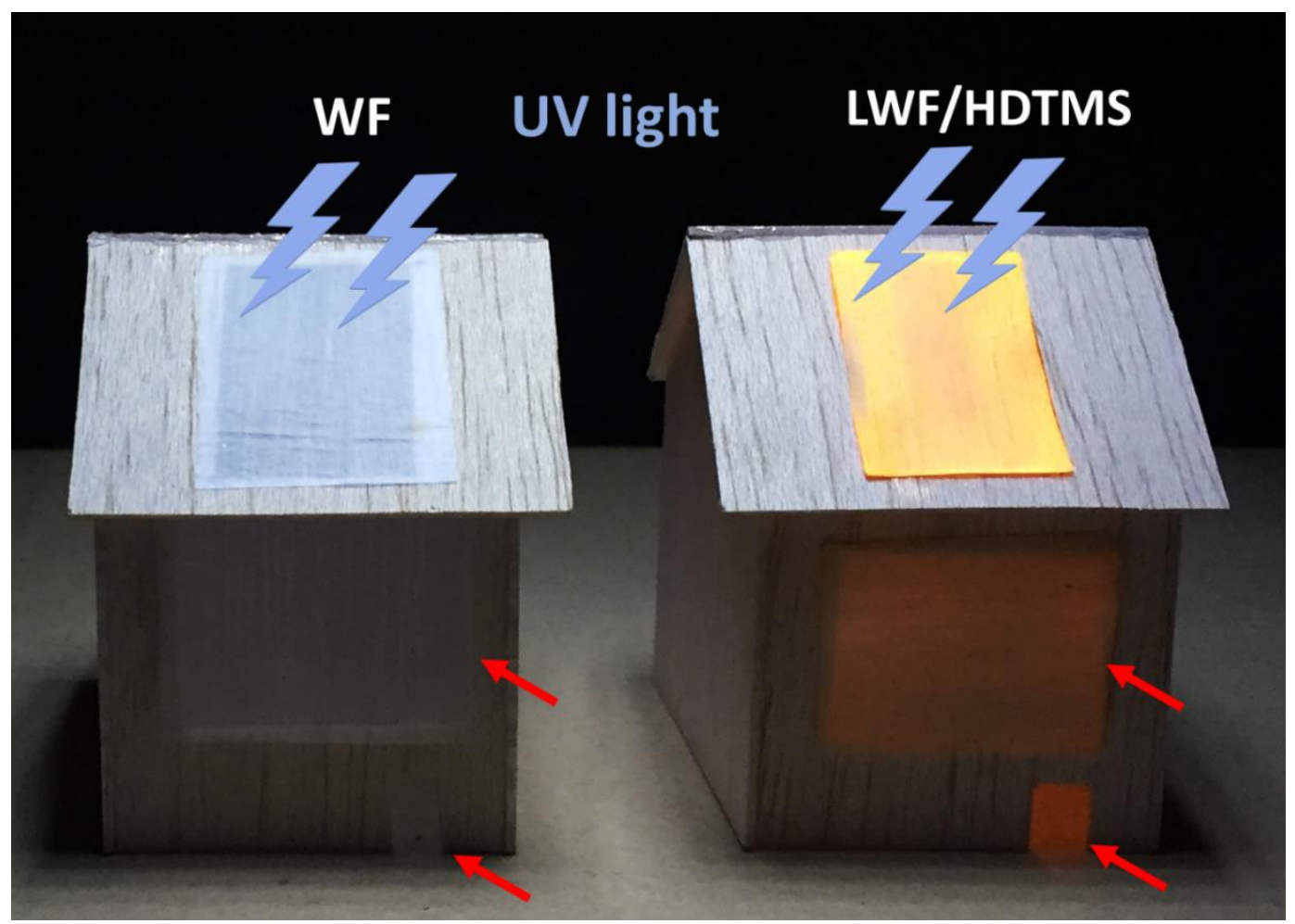

Figure S26. A demonstration of a uniform illumination using LWF/HDTMS panel as a lighting material when exposed to a UV light source. The red arrows indicate dark (left) and illuminated (right) spaces when using WF and LWF/HDTMS, respectively. 


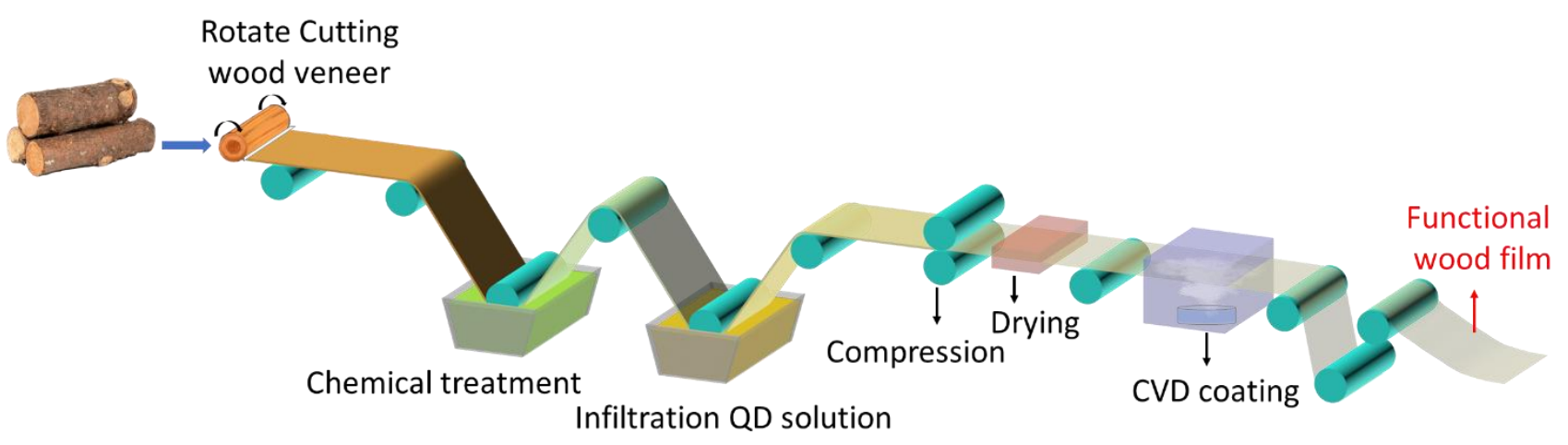

Figure S27. Potential engineering production development of functional wood film for optical applications using continues roll-to-roll system. 


\section{References}

(1) Fu, Q.; Yan, M.; Jungstedt, E.; Yang, X.; Li, Y.; Berglund, L. A. Transparent Plywood as a Load-Bearing and Luminescent Biocomposite. Compos. Sci. Technol. 2018, 164, 296303.

(2) Li, Y.; Fu, Q.; Yu, S.; Yan, M.; Berglund, L. Optically Transparent Wood from a Nanoporous Cellulosic Template: Combining Functional and Structural Performance. Biomacromolecules 2016, 17, 1358-1364.

(3) Brinkmann, M.; Hayden, J.; Letz, M.; Reichel, S.; Click, C.; Mannstadt, W.; Schreder, B.; Wolff, S.; Ritter, S.; Davis, M. J.; Bauer, T.; Ren, H.; Fan, Y. H.; Wu, S. T.; Bonrad, K.; Krätzig, E.; Buse, K.; Paquin, Roger. Optical Materials and Their Properties. In Springer Handbook of Lasers and Optics; Springer Berlin Heidelberg: Berlin, Heidelberg, 2012; pp 253-399.

(4) Sharma, C. P. Engineering Materials: Properties and Applications of Metals and Alloys; PHI Learning Pvt. Ltd.: Delhi, 2003.

(5) Springer, H.; Baron, C.; Szczepaniak, A.; Uhlenwinkel, V.; Raabe, D. Stiff, Light, Strong and Ductile: Nano-Structured High Modulus Steel. Sci. Rep. 2017, 7, 17-22.

(6) Dursun, T.; Soutis, C. Recent Developments in Advanced Aircraft Aluminium Alloys. Mater. Des. 2014, 56, 862-871.

(7) Gludovatz, B.; Hohenwarter, A.; Catoor, D.; Chang, E. H.; George, E. P.; Ritchie, R. O. A Fracture-Resistant High-Entropy Alloy for Cryogenic Applications. Science 2014, 345, $1153-1158$.

(8) Kim, S.-H.; Kim, H.; Kim, N. J. Brittle Intermetallic Compound Makes Ultrastrong LowDensity Steel with Large Ductility. Nature 2015, 518, 77-79.

(9) Crompton, T. R. Physical Testing of Plastics; Smithers Rapra Technology Ltd.: Shropshire, 2012; pp 1-148.

(10) Henriksson, M.; Berglund, L. A.; Isaksson, P.; Lindström, T.; Nishino, T. Cellulose 
Nanopaper Structures of High Toughness. Biomacromolecules 2008, 9, 1579-1585.

(11) Nogi, M.; Iwamoto, S.; Nakagaito, A. N.; Yano, H. Optically Transparent Nanofiber Paper. Adv. Mater. 2009, 21, 1595-1598.

(12) Ansari, F.; Galland, S.; Johansson, M.; Plummer, C. J. G.; Berglund, L. A. Cellulose Nanofiber Network for Moisture Stable, Strong and Ductile Biocomposites and Increased Epoxy Curing Rate. Composites, Part A 2014, 63, 35-44.

(13) Tang, H.; Butchosa, N.; Zhou, Q. A Transparent, Hazy, and Strong Macroscopic Ribbon of Oriented Cellulose Nanofibrils Bearing Poly(ethylene Glycol). Adv. Mater. 2015, 27, 2070-2076.

(14) Wang, B.; Torres-Rendon, J. G.; Yu, J.; Zhang, Y.; Walther, A. Aligned Bioinspired Cellulose Nanocrystal-Based Nanocomposites with Synergetic Mechanical Properties and Improved Hygromechanical Performance. ACS Appl. Mater. Interfaces 2015, 7, 45954607.

(15) Sehaqui, H.; Ezekiel Mushi, N.; Morimune, S.; Salajkova, M.; Nishino, T.; Berglund, L. A. Cellulose Nanofiber Orientation in Nanopaper and Nanocomposites by Cold Drawing. ACS Appl. Mater. Interfaces 2012, 4, 1043-1049.

(16) Torres-Rendon, J. G.; Schacher, F. H.; Ifuku, S.; Walther, A. Mechanical Performance of Macrofibers of Cellulose and Chitin Nanofibrils Aligned by Wet-Stretching: A Critical Comparison. Biomacromolecules 2014, 15, 2709-2717.

(17) Yao, J.; Chen, S.; Chen, Y.; Wang, B.; Pei, Q.; Wang, H. Macrofibers with High Mechanical Performance Based on Aligned Bacterial Cellulose Nanofibers. ACS Appl. Mater. Interfaces 2017, 9, 20330-20339.

(18) Wang, S.; Jiang, F.; Xu, X.; Kuang, Y.; Fu, K.; Hitz, E.; Hu, L. Super-Strong, Super-Stiff Macrofibers with Aligned, Long Bacterial Cellulose Nanofibers. Adv. Mater. 2017, 29, 1702498.

(19) Håkansson, K. M. O.; Fall, A. B.; Lundell, F.; Yu, S.; Krywka, C.; Roth, S. V.; Santoro, 
G.; Kvick, M.; Prahl Wittberg, L.; Wågberg, L.; Söderberg, D. Hydrodynamic Alignment and Assembly of Nanofibrils Resulting in Strong Cellulose Filaments. Nat. Commun. 2014, 5, 4018 .

(20) Mittal, N.; Ansari, F.; Gowda.V, K.; Brouzet, C.; Chen, P.; Larsson, P. T.; Roth, S. V.; Lundell, F.; Wågberg, L.; Kotov, N. A.; Söderberg, L. D. Multiscale Control of Nanocellulose Assembly: Transferring Remarkable Nanoscale Fibril Mechanics to Macroscale Fibers. ACS Nano 2018, 12, 6378-6388.

(21) Mittal, N.; Benselfelt, T.; Ansari, F.; Gordeyeva, K.; Roth, S. V; Wågberg, L.; Söderberg, L. D. Ion-Specific Assembly of Strong, Tough, and Stiff Biofibers. Angew. Chem., Int. Ed. 2019, 58, 18562-18569.

(22) Zhu, M.; Wang, Y.; Zhu, S.; Xu, L.; Jia, C.; Dai, J.; Song, J.; Yao, Y.; Wang, Y.; Li, Y.; Henderson, D.; Luo, W.; Li, H.; Minus, M. L.; Li, T.; Hu, L. Anisotropic, Transparent Films with Aligned Cellulose Nanofibers. Adv. Mater. 2017, 29, 1606284.

(23) Song, J.; Chen, C.; Zhu, S.; Zhu, M.; Dai, J.; Ray, U.; Li, Y.; Kuang, Y.; Li, Y.; Quispe, N.; Yao, Y.; Gong, A.; Leiste, U. H.; Bruck, H. A.; Zhu, J. Y.; Vellore, A.; Li, H.; Minus, M. L.; Jia, Z.; Martini, A.; et al. Processing Bulk Natural Wood into a High-Performance Structural Material. Nature 2018, 554, 224-228.

(24) Li, T.; Zhai, Y.; He, S.; Gan, W.; Wei, Z.; Heidarinejad, M.; Dalgo, D.; Mi, R.; Zhao, X.; Song, J.; Dai, J.; Chen, C.; Aili, A.; Vellore, A.; Martini, A.; Yang, R.; Srebric, J.; Yin, X.; Hu, L. A Radiative Cooling Structural Material. Science 2019, 364, 760-763.

(25) Frey, M.; Biffi, G.; Adobes-Vidal, M.; Zirkelbach, M.; Wang, Y.; Tu, K.; Hirt, A. M.; Masania, K.; Burgert, I.; Keplinger, T. Tunable Wood by Reversible Interlocking and Bioinspired Mechanical Gradients. Adv. Sci. 2019, 6, 1802190.

(26) Frey, M.; Widner, D.; Segmehl, J. S.; Casdorff, K.; Keplinger, T.; Burgert, I. Delignified and Densified Cellulose Bulk Materials with Excellent Tensile Properties for Sustainable Engineering. ACS Appl. Mater. Interfaces 2018, 10, 5030-5037. 
(27) Liu, Y.; Li, B.; Mao, W.; Hu, W.; Chen, G.; Liu, Y.; Fang, Z. Strong Cellulose-Based Materials by Coupling Sodium Hydroxide-Anthraquinone (NaOH-AQ) Pulping with Hot Pressing from Wood. ACS Omega 2019, 4, 7861-7865.

(28) Yano, H. Potential Strength for Resin-Impregnated Compressed Wood. J. Mater. Sci. Lett. 2001, 20, 1127-1129.

(29) Shams, M. I.; Yano, H.; Endou, K. Compressive Deformation of Wood Impregnated with Low Molecular Weight Phenol Formaldehyde (PF) Resin I: Effects of Pressing Pressure and Pressure Holding. J. Wood Sci. 2004, 50, 337-342.

(30) Frey, M.; Schneider, L.; Masania, K.; Keplinger, T.; Burgert, I. Delignified WoodPolymer Interpenetrating Composites Exceeding the Rule of Mixtures. ACS Appl. Mater. Interfaces 2019, 11, 35305-35311.

(31) Zhu, M.; Song, J.; Li, T.; Gong, A.; Wang, Y.; Dai, J.; Yao, Y.; Luo, W.; Henderson, D.; Hu, L. Highly Anisotropic, Highly Transparent Wood Composites. Adv. Mater. 2016, 28, $5181-5187$.

(32) Li, Y.; Fu, Q.; Yang, X.; Berglund, L. Transparent Wood for Functional and Structural Applications. Philos. Trans. R. Soc., A 2018, 376, 20170182.

(33) Han, X.; Ye, Y.; Lam, F.; Pu, J.; Jiang, F. Hydrogen-Bonding-Induced Assembly of Aligned Cellulose Nanofibers into Ultrastrong and Tough Bulk Materials. J. Mater. Chem. A 2019, 7, 27023-27031.

(34) Khakalo, A.; Tanaka, A.; Korpela, A.; Hauru, L. K. J.; Orelma, H. All-Wood Composite Material by Partial Fiber Surface Dissolution with an Ionic Liquid. ACS Sustain. Chem. Eng. 2019, 7, 3195-3202.

(35) Gan, W.; Chen, C.; Kim, H.-T.; Lin, Z.; Dai, J.; Dong, Z.; Zhou, Z.; Ping, W.; He, S.; Xiao, S.; Yu, M.; Hu, L. Single-Digit-Micrometer Thickness Wood Speaker. Nat. Commun. 2019, 10, 5084.

(36) Li, X.; Tabil, L. G.; Panigrahi, S. Chemical Treatments of Natural Fiber for Use in Natural 
Fiber-Reinforced Composites: A Review. J. Polym. Environ. 2007, 15, 25-33.

(37) Ugbolue, S. C. O. Structure/Property Relationships in Textile Fibres. Text. Prog. 1990, $20,1-43$.

(38) Li, Z.; Chen, C.; Mi, R.; Gan, W.; Dai, J.; Jiao, M.; Xie, H.; Yao, Y.; Xiao, S.; Hu, L. A Strong, Tough, and Scalable Structural Material from Fast-Growing Bamboo. Adv. Mater. 2020, 32, 1906308.

(39) Li, K.; Wang, S.; Chen, H.; Yang, X.; Berglund, L. A.; Zhou, Q. Self - Densification of Highly Mesoporous Wood Structure into a Strong and Transparent Film. Adv. Mater. 2020, 2003653. 